\title{
Ligand-Induced Conformational Rearrangements Promote Interaction between the Escherichia coli Enterobactin Biosynthetic Proteins EntE and EntB ${ }^{\dagger}$
}

\footnotetext{
${ }^{\dagger}$ This work was supported by Discovery Grant 341983-07 from the Natural Sciences and Engineering Research Council of Canada to PDP
}

\author{
Sofia Khalil and Peter D. Pawelek ${ }^{*}$ \\ Department of Chemistry and Biochemistry, Concordia University, \\ 7141 Sherbrooke St., W., Montreal, Quebec, Canada, H4B 1R6
}

*Correspondence should be addressed to: Peter D. Pawelek, Tel: 514-848-2424 ext. 3118; Fax:

514-848-2868; E-mail: ppawelek@alcor.concordia.ca

Running title: Efficient EntE-EntB interaction requires 2,3-dihydroxybenzoic acid 


\begin{abstract}
Abbreviations
The following abbreviations are used in this manuscript: 2,3-DHB: 2,3-dihydroxybenzoic acid; 2,5-DHB: 2,5-dihydroxybenzoic acid; 3,5-DHB: 3,5-dihydroxybenzoic acid; AMP: adenosine monophosphate; ArCP: aryl carrier protein; ATP: adenosine triphosphate; CD: circular dichroism; DTT: dithiothreitol; FRET: fluorescence resonance energy transfer; H6-EntB: purified, recombinant hexahistidine-tagged E. coli EntB; H6-EntE: purified, recombinant hexahistidinetagged E. coli EntE; ICL: isochorismate lyase; ITC: isothermal titration calorimetry; NRPS: nonribosomal peptide synthesis; PDB: Protein Data Bank; RMSD: root mean square deviation; SDSPAGE: sodium dodecyl sulfate polyacrylamide gel electrophoresis; TCEP: tris(2carboxyethyl)phosphine.
\end{abstract}




\section{Abstract}

Siderophores are small-molecule iron chelators that many bacteria synthesize and secrete in order to survive in iron-depleted environments. Biosynthesis of enterobactin, the $E$. coli catecholate siderophore, requires adenylation of 2,3-DHB by the cytoplasmic enzyme EntE. The DHBAMP product is then transferred to the active site of holo-EntB subsequent to formation of an EntE-EntB complex. Here we investigate the binding of 2,3-DHB to EntE, and how DHB binding affects EntE-EntB interaction. We overexpressed and purified recombinant forms of EntE and EntB with N-terminal hexahistidine tags (H6-EntE and H6-EntB). Isothermal titration calorimetry showed that 2,3-DHB binds to H6-EntE with a 1:1 stoichiometry and a $\mathrm{K}_{\mathrm{D}}$ of $7.4 \mu \mathrm{M}$. Fluorescence spectra revealed enhanced 2,3-DHB emission at $440 \mathrm{~nm}\left(\lambda_{\mathrm{ex}}=280 \mathrm{~nm}\right)$ when bound to H6-EntE due to fluorescence resonance energy transfer (FRET) between EntE intrinsic fluorophore donors and the bound 2,3-DHB acceptor. A FRET signal was not observed when H6-EntE was mixed with either 2,5-DHB or 3,5-DHB. The H6-EntE-2,3-DHB FRET signal was quenched by $H 6$-EntB in a concentration-dependent manner. From these data we were able to determine the $\mathrm{EC}_{50}$ of EntE-EntB interaction to be approximately $1.5 \mu \mathrm{M}$. We also found by fluorescence and $\mathrm{CD}$ measurements that $H 6$-EntB can bind 2,3-DHB, resulting in conformational changes in the protein. Additional alterations in the H6-EntB near-UV and far-UV CD spectra were observed upon mixture with H6-EntE and 2,3-DHB, suggesting that further conformational rearrangements occur in EntB upon interaction with substrate-loaded EntE. We also found that H6-EntB as a bait protein pulled down a higher concentration of chromosomally-expressed EntE in the presence of exogenous 2,3-DHB. Taken together, our results show that binding of 2,3DHB to EntE and EntB primes these proteins for efficient complexation, thus facilitating direct channeling of the siderophore precursor 2,3-DHB-AMP. 
Keywords: siderophore, enterobactin, protein-protein interactions, FRET, substrate channeling

\section{Introduction}

Iron is an essential element for most bacteria since it is used for many cellular processes related to metabolism and signaling. ${ }^{1}$ In order to survive in iron-depleted environments, many bacteria synthesize and secrete iron-chelating molecules known as siderophores that have high $\mathrm{Fe}^{3+}$-binding affinities. Siderophores, and the proteins involved in their synthesis, secretion, modification, and uptake, are considered virulence factors in a number of bacterial pathogens. ${ }^{2}$ Escherichia coli K12 synthesizes and secretes a single catecholate siderophore called enterobactin, which binds $\mathrm{Fe}^{3+}$ with a $\mathrm{K}_{\mathrm{A}}$ of approximately $10^{50} \mathrm{M}^{-1} .{ }^{3}$ Upon secretion, enterobactin chelates bioavailable $\mathrm{Fe}^{3+}$ from the extracellular environment. Ferric enterobactin is then bound by the E. coli outer membrane transporter FepA and imported by a TonB-dependent uptake system. ${ }^{4}$ The ferric siderophore is translocated through the cell envelope and subsequently degraded by the Fes esterase in order to liberate the iron for various cellular processes. It has recently been reported that extraintestinal strains of E. coli, including uropathogenic E. coli (UPEC), possess a five-gene cluster known as iro $A$ that is responsible for modifying enterobactin to enhance its virulence. ${ }^{5,6}$ Understanding the molecular mechanisms by which enterobactin is synthesized and secreted by $E$. coli is thus of high priority since such mechanisms may reveal novel antimicrobial targets.

Enterobactin is synthesized in the E. coli cytoplasm by seven enzymes: EntC, EntB, EntA, EntE, EntF, EntD, and EntH. The five enzymes directly involved in enterobactin biosynthesis can be grouped into two functional modules: the DHB module (EntC, EntB (N-terminal domain), and EntA) and the non-ribosomal peptide synthesis (NRPS) module (EntE, EntB (C- 
terminal domain), and EntF). In the DHB module, chorismate is converted to 2,3-DHB through the sequential activities of EntC, EntB, and EntA. ${ }^{7-11}$ The DHB module functions upstream of the NRPS module, and the two are intrinsically linked through the involvement of the bifunctional EntB protein, in which the N-terminal isochorismate lyase (ICL) domain participates in DHB synthesis while the C-terminal aryl carrier protein (ArCP) domain participates in NRPS. Enterobactin is ultimately produced during NRPS by the condensation of three molecules of 2,3dihydroxybenzoate (2,3-DHB) and three molecules of L-serine yielding the tricatecholate ester. The enzyme EntD catalyzes the phosphopantetheinylation of the ArCP domains of EntB and EntF. ${ }^{12,13}$ EntH is a proofreading activity that corrects NRPS misacylation events. ${ }^{14-16}$

The enzyme EntE catalyzes the adenylation of 2,3-DHB produced by EntA, thus activating it for subsequent steps in the NRPS module (Fig. 1). EntE has a subunit size of $59 \mathrm{kDa}$ and is reported to be monomeric. ${ }^{17}$ The EntE product, 2,3-DHB-AMP, is directly transferred to the phosphopantetheine cofactor anchored at the S245 residue in the active site of holo-EntB. ${ }^{12}$ Experiments have shown that the turnover number of EntE increases approximately 10-fold in the presence of holo-EntB. ${ }^{18}$ In the absence of holo-EntB, release of DHB-AMP from the EntE active site is slow, due to the high affinity of binding of the product to the enzyme. Site-directed mutagenesis of residues hypothesized to be at the interaction interface of the EntE - holo-EntB complex was reported to have an effect on EntE catalytic activity. ${ }^{19}$

Although no three-dimensional structure of EntE is currently available, crystal structures of DhbE, a homologous protein involved in the biosynthesis of the B. subtilis catecholate siderophore bacillibactin, provide us with some insights into the EntE active site. DhbE has been crystallized in three forms: $(i)$ the apo form (PDB code: 1mdf), (ii) with 2,3-DHB-adenylate bound (PDB code: $1 \mathrm{mdb}$ ), and (iii) with 2,3-DHB and AMP bound (PDB code: $1 \mathrm{md} 9){ }^{20}$ In the sub- 
strate-bound structures, 2,3-DHB binds at the floor of a hydrophobic cavity and is stabilized by the active site residue DhbE Y236. The carboxylate moiety of 2,3-DHB is further stabilized by interactions with DhbE residues H234 and K517. The ATP cofactor binds near the entrance of the cavity, proximal to a loop known as the 'P-loop', which closes over the active site during catalysis. Comparison of the apo- and holo-DhbE structures indicates that the protein undergoes local conformational changes (i.e, loop movements), but no gross structural rearrangements to the overall fold, upon substrate binding.

EntB is the sequentially-related enterobactin biosynthetic enzyme immediately downstream of EntE. The crystal structure of EntB has been reported. ${ }^{19}$ The $32.5 \mathrm{kDa}$ EntB subunit is composed of two discrete and independently folded domains: an N-terminal isochorismate lyase (ICL) domain (residues 26-208), and a C-terminal aryl carrier protein (ArCP) domain (residues 214-285). Crystallographic data revealed an EntB dimer in the asymmetric unit, with the majority of the inter-subunit interface occurring between ICL domains in the dimer. The ICL domain comprises most of the subunit mass (183 residues out of 285 residues in the subunit). Mutagenesis studies revealed that alteration of residues in the ArCP domain affected stimulation of EntE activity by EntB, suggesting their role in the interaction interface. ${ }^{19}$ Thus, there is well established, albeit indirect, evidence for an interaction between EntE and EntB.

Here we investigate the nature of EntE substrate binding and its effects on the EntE-EntB protein-protein interaction. Isothermal titration calorimetry has provided us with unambiguous equilibrium dissociation constant describing binding of 2,3-DHB substrate to EntE. Furthermore, we show that 2,3-DHB can act as a fluorescent sensor of the EntE active site. We exploited this phenomenon to directly measure the EntE-EntB interaction. Our circular dichroism studies and pull-down assays support our fluorescence binding data and show that the formation of an EntE- 
EntB complex is most efficient in the presence of 2,3-DHB. Our experimental approaches have provided the first direct measurement of the EntE-EntB interaction, and have generated insights into how protein-protein interactions in the enterobactin biosynthetic machinery may be essential in modulating substrate flux that would result in maximal siderophore production while minimizing deleterious consequences of intracellular accumulation of intermediates.

\section{Results}

\section{Homology modeling of $E$. coli EntE predicts positions of ligand-binding residues and in-} trinsic fluorophores

In order to gain insights into the nature of substrate binding to E. coli EntE, as well as the spatial distribution of intrinsic fluorophore residues (Tyr, Trp) throughout the protein, we generated a homology-based model using the structure of the $B$. subtilis bacillibactin biosynthetic enzyme DhbE (PDB code: $1 \mathrm{md} 9$ ) as a template. These enzymes share $46.5 \%$ sequence identity (Fig. 2a). The structure-based alignment obtained from the FFAS03 server was used as an input for Modeller 9v3, which produced a three-dimensional model of E. coli EntE as output. The EntE model superimposes well with the DhbE structure, with an overall RMSD of $0.271 \AA$ (Fig. 2b). The stereochemistry of the model was analyzed using the VADAR suite. ${ }^{21}$ Ramachandran analysis showed that $90 \%$ of the model's residues are in the core region, $47 \%$ in the allowed region, $1 \%$ in the generous region, and no residues were found in the disallowed region. The overall general distribution of the intrinsic fluorophores in the model is such that most of the tyrosine residues populate the interior of the protein whereas most tryptophan residues are surface-exposed. This distribution is consistent with the reported DhbE structure. ${ }^{20}$ We computationally introduced a 2,3-DHB molecule into the active site of our EntE model by using the 2,3-DHB coordinates 
found in the DhbE active site (Fig. 2c). The 2,3-DHB molecule was introduced using the same rotation and translation matrices determined by LSQMAN for optimal EntE-DhbE superposition. Five tyrosine residues were found to be most proximal (distance $<10 \AA$ ) to the DHB substrate: Y207, Y226, Y236, Y341, and Y389. All five tryptophan residues in the EntE model were found to be distal (> $15 \AA)$ to the active site. Four of the five tryptophans in the model are surfaceexposed. The tryptophan most proximal to the modeled DHB position, W286 (distance =15.6 $\AA$ ), was the only buried tryptophan found in the model. The model also identified EntE residues that could potentially bind to non-aromatic portions of 2,3-DHB. Residues K519 and H233 are within hydrogen bonding distance of the carboxylate moiety of the substrate and superimpose with DhbE residues previously identified from the crystal structure as stabilizing the carboxylate of bound 2,3-DHB (Fig. 2d). ${ }^{20}$ According to our model, EntE residues N234 and S239 are within hydrogen bonding distance of the 2-hydroxyl and 3-hydroxyl groups of 2,3-DHB, respectively. These residues superimpose with asparagine and serine residues binding corresponding DHB OH groups in the substrate-bound DhbE crystal structure.

\section{EntE has a single, micromolar-affinity binding site for its substrate 2,3-DHB}

We used isothermal titration calorimetry (ITC) to directly measure the binding of 2,3-DHB to purified recombinant hexahistidine-tagged EntE (H6-EntE). 2,3-DHB at a concentration of 500 $\mu \mathrm{M}$ was injected into a $50 \mu \mathrm{M}$ solution of $H 6$-EntE and heats of binding were measured over 24 injections (Fig. 3, upper panel). Integrated heats of injection of $500 \mu \mathrm{M}$ 2,3-DHB into buffer were subtracted from the integrated heats of ligand binding to EntE. The corrected heats were plotted as a function of the molar ratio of DHB to H6-EntE (Fig. 3, lower panel). These data fit well to the single-site binding equation in Origin 5.0, indicating that 2,3-DHB binds to H6-EntE 
with a stoichiometry of $0.92 \pm 0.01$, and with an equilibrium dissociation constant $\left(\mathrm{K}_{\mathrm{D}}\right)$ of $7.35 \pm$ $0.31 \mu \mathrm{M}$. Our ITC data revealed the $\Delta \mathrm{H}$ of 2,3-DHB binding to $H 6$-EntE to be $-13.46 \pm 0.15$ $\mathrm{kcal} / \mathrm{mol}$ and the $\mathrm{T} \Delta \mathrm{S}$ to be $-7.03 \mathrm{kcal} / \mathrm{mol}$, such that the overall $\Delta \mathrm{G}$ of the binding reaction was $-6.43 \mathrm{kcal} / \mathrm{mol}$.

\section{Binding of 2,3-DHB to EntE results in a substrate-specific FRET signal}

We obtained H6-EntE fluorescence emission spectra in the presence and absence of 2,3-DHB substrate. Analysis of H6-EntE fluorescence emission upon excitation at $280 \mathrm{~nm}$ revealed a single emission peak at $330 \mathrm{~nm}$ (Fig. 4a, black circles). Upon addition of $50 \mu \mathrm{M}$ 2,3-DHB to $3 \mu \mathrm{M}$ H6-EntE, we observed a decrease in the $330 \mathrm{~nm}$ emission peak along with the appearance of a new emission peak at $440 \mathrm{~nm}$ (Fig. 4a, green circles). This peak coincides with the fluorescence emission maximum of 2,3-DHB (Fig. 4a, magenta circles), although in the absence of protein the fluorescence intensity of an equivalent concentration $(50 \mu \mathrm{M})$ of the substrate was markedly lower. The spectral overlap between tyrosine emission and 2,3-DHB excitation maxima suggests that the increase in 2,3-DHB fluorescence intensity at $440 \mathrm{~nm}$ in the presence of H6-EntE is due to fluorescence resonance energy transfer (FRET). Fluorescence excitation spectra $\left(\lambda_{\mathrm{em}}=440\right.$ $\mathrm{nm}$ ) of 2,3-DHB solutions in the presence and absence of H6-EntE indicated that the excitation maxima of 2,3-DHB were not significantly altered upon binding to H6-EntE, and that a prominent peak occurred around $280 \mathrm{~nm}$ in the presence of H6-EntE (Fig. 4b). Fluorescence emission spectra were also collected at $275 \mathrm{~nm}$, a wavelength that is closer to that of maximal tyrosine excitation and further from that of maximal tryptophan excitation. Excitation at $275 \mathrm{~nm}$ was observed to increase the intensity of the emission peak at $440 \mathrm{~nm}$ relative to excitation at $280 \mathrm{~nm}$ (data not shown). The addition of increasing concentrations of 2,3-DHB $(5 \mu \mathrm{M}-80 \mu \mathrm{M})$ to a 
fixed concentration of H6-EntE ( $3 \mu \mathrm{M})$ resulted in concentration-dependent increases in fluorescence intensity at $440 \mathrm{~nm}$ with corresponding decreases in fluorescence intensity at $330 \mathrm{~nm}$. These concentration-dependent changes in fluorescence intensity reflect 2,3-DHB binding to H6EntE since the data fit well to hyperbolic plots describing single-site saturable binding (Figs. 4c and $4 \mathrm{~d}$ ). Hyperbolic fits are consistent with $\mathrm{EC}_{50}$ values of $12.9 \mu \mathrm{M}$ (obtained from the increase in fluorescence intensity at $440 \mathrm{~nm}$; Fig. 4c) and $9.2 \mu \mathrm{M}$ (obtained from the quenching of fluorescence intensity at $330 \mathrm{~nm}$; Fig. 4d).

We investigated the specificity of binding of DHB isomers to H6-EntE and recombinant hexahistidine-tagged E. coli EntB (H6-EntB). Fluorescence emission spectra were collected for H6-EntB and H6-EntE in the presence of equivalent concentrations of 2,3-DHB, 2,5-DHB and 3,5-DHB. We found that the $H 6$-EntB emission peak at $330 \mathrm{~nm}$ (Fig. 5a, black circles) was maximally quenched in the presence of 2,3-DHB (Fig. 5a, green circles). We also found that H6EntB intrinsic fluorescence was quenched upon addition of 2,5-DHB (Fig. 5a, red circles) and 3,5-DHB (Fig. 5a, blue circles), albeit to a lesser extent. When solutions of H6-EntE were mixed with the DHB isomers, we found that only 2,3-DHB could quench intrinsic H6-EntE fluorescence (Fig. 5b). Furthermore, we only observed a $440 \mathrm{~nm}$ emission peak when $H 6$-EntE was mixed with $50 \mu \mathrm{M}$ 2,3-DHB. No quenching of H6-EntE intrinsic fluorescence was observed upon addition of equivalent concentrations of 2,5-DHB (Fig. 5b, red circles) or 3,5-DHB (Fig. 5B, blue circles); furthermore, neither 2,5-DHB nor 3,5-DHB exhibited a $440 \mathrm{~nm}$ emission peak in the presence of $H 6$-EntE upon excitation at $280 \mathrm{~nm}$ even though both of these DHB isomers also exhibit fluorescence emission maxima at $440 \mathrm{~nm}$ (data not shown). 


\section{Binding of 2,3-DHB to both EntE and EntB results in conformational changes that promote formation of a transient EntE-EntB complex}

We investigated the effect of EntB-EntE interaction on 2,3-DHB binding to H6-EntE using our fluorescence assay. We found that $H 6$-EntB quenched $440 \mathrm{~nm}$ fluorescence emission of H6-EntE-bound 2,3-DHB in a concentration-dependent manner (Fig. 6). Half-maximal quenching of H6-EntE-bound 2,3-DHB by H6-EntB indicates that the $\mathrm{EC}_{50}$ of the EntB-EntE interaction is $1.5 \mu \mathrm{M}$, assuming that the observed decrease in fluorescence is due to the EntBEntE interaction affecting the positioning of 2,3-DHB binding in the H6-EntE active site and thus its ability to fluoresce at $440 \mathrm{~nm}$. Circular dichroism (CD) was used to measure protein structural changes occurring upon mixture of $H 6$-EntE and H6-EntB in the presence or absence of 2,3-DHB. All spectra in which proteins were mixed with 2,3-DHB were corrected for ellipticity signal contributions from 2,3-DHB in the absence of protein. Far-UV CD spectra revealed that both H6-EntE (Fig. 7a, blue circles) and H6-EntB (Fig. 7a, red circles) in solution exhibited similar major ellipticity minima at $210 \mathrm{~nm}$ and $222 \mathrm{~nm}$. The addition of a saturating concentration of 2,3-DHB to H6-EntE (Fig. 7b, blue circles) did not significantly alter its far-UV CD spectrum, indicating no large changes in secondary structure occurred upon substrate binding. A decrease in the ellipticity minima in the H6-EntB spectrum was observed upon the addition of 2,3-DHB (Fig. 7b, blue circles). Far-UV spectra were also obtained for mixtures of H6EntE and H6-EntB in the presence and absence of DHB. Spectra of the $\{H 6-$ EntE $+H 6-E n t B\}$ mixtures in which the H6-EntB signals were subtracted revealed no significant changes in H6EntE far-UV spectra in the presence or absence of 2,3-DHB (Fig. 7a and 7b, cyan circles). In contrast, a spectrum of the $\{H 6$-EntE $+H 6$-EntB $\}+$ DHB mixture in which the H6-EntE signal was subtracted revealed a significant change in H6-EntB far-UV spectrum (Fig. 7b, magenta cir- 
cles). The near-UV CD spectrum of H6-EntE in the absence of 2,3-DHB (Fig. 7c, blue circles) indicates a dominant positive peak centered between $270 \mathrm{~nm}$ and $280 \mathrm{~nm}$, indicative of contributions from tyrosine residues. This spectrum did not change when H6-EntE was mixed with 2,3DHB (Fig. 7d, blue circles). The H6-EntB near-UV -DHB spectrum (Fig. 7c, red circles) revealed two minor positive peaks at $255 \mathrm{~nm}$ and $277 \mathrm{~nm}$, and a major negative peak at $290 \mathrm{~nm}$. In the presence of 2,3-DHB, the H6-EntB near-UV spectrum exhibits a similar overall shape, but with altered peak magnitudes compared to the-DHB spectrum (Fig. 7d, red circles). As with our far-UV experiments, we collected near-UV spectra of $\{H 6-E n t E+H 6-E n t B\}$ mixtures in the presence and absence of 2,3-DHB. The spectrum of the $\{H 6-\mathrm{EntE}+H 6-\mathrm{EntB}\}-\mathrm{DHB}$ mixture in which the H6-EntB signal was subtracted (Fig. 7c, cyan circles) revealed no major change relative to the H6-EntE spectrum (Fig. 7c, blue circles). We observed a small reduction in overall ellipticity of the $\{H 6$-EntE $+H 6$-EntB $\}+$ DHB spectrum after subtraction of the H6-EntB signal (Fig. 7d, cyan circles) when compared to the H6-EntE spectrum (Fig. 7d, blue circles). In contrast, large changes in the peak amplitudes at $255 \mathrm{~nm}$ and $277 \mathrm{~nm}$ were observed in a spectrum of the $\{H 6$-EntE $+H 6$-EntB $\}$ mixture in which the H6-EntE signal was subtracted (Fig. 7 d, magenta circles) compared to the H6-EntB +DHB spectrum obtained in the absence of H6-EntE (Fig. 7d, red circles).

\section{H6-EntB bait protein efficiently pulls down chromosomally-expressed $E$. coli EntE only in}

\section{the presence of exogenous 2,3-DHB}

To investigate the ability of our recombinantly expressed $H 6$-EntB to form complexes with $E$. coli partner proteins in situ, we used H6-EntB as a bait protein to pull down chromosomallyexpressed interacting partners from lysates of $E$. coli cells grown in M63 minimal media in the 
presence of the iron chelator 2,2'-dipyridyl. This iron-depleted media condition was employed in order to induce expression of chromosomally-encoded enterobactin biosynthetic proteins by derepression of Fur. Pull down assays were achieved by binding H6-EntB bait protein to $\mathrm{Co}^{2+}$ chelate resin, flowing over cell lysates, and gently washing away unbound proteins. When exposed to lysates pre-incubated with exogenous 2,3-DHB, the H6-EntB bait protein was able to pull down a major protein band migrating at approximately $60 \mathrm{kDa}$ as determined by SDS-PAGE analysis (Fig. 8, band 'd'), which corresponds to the molecular weight of E. coli EntE. In the absence of exogenous 2,3-DHB, a minor band migrating at this position was observed to be pulled down (Fig. 8, lane -DHB, band 'a'). Bands migrating at approximately $34 \mathrm{kDa}$, the expected molecular weight of H6-EntB, were observed +/- exogenous 2,3-DHB (Fig. 8, bands 'b' and 'e'). Below this band, we also observed a second major band migrating at approximately $25 \mathrm{kDa}$ (Fig. 8 , bands ' $c$ ' and ' $\mathrm{f}$ ').

We were able to perform mass spectrometry analysis on tryptic peptides recovered from the proteins corresponding to bands ' $b$-' $\mathrm{f}$ '; all other bands contained insufficient amounts of protein for this analysis. Table 1 shows the peptides recovered from bands ' $\mathrm{d}$ ', 'e', and ' $\mathrm{f}$ ' shown in Figure 8; all of these peptides were obtained from lysates spiked with exogenous 2,3-DHB prior to pull-down. Peptides corresponding to EntE in band 'd' (Table 1, rows 3-21) represent 57\% total EntE sequence coverage. Peptides corresponding to EntB in band 'e' (Table 1, rows 22-37) represent $66 \%$ total EntB sequence coverage. Peptides corresponding to EntB in band ' $\mathrm{f}$ ' (Table 1, rows 38-41) represent $18 \%$ total EntB sequence coverage. Peptides were also recovered from bands 'a'-'c' obtained from -DHB samples (data not shown). In the absence of exogenous 2,3DHB, no peptides corresponding to EntE could be identified by mass spectrometry, although the silver-stained gel shows a faint band (Fig. 8, band 'a') at its expected position. The low amount 
of protein in this band likely resulted in poor peptide recovery such that any EntE signals were below the sensitivity threshold of the mass spectrometer. Mass spectrometry data of peptides recovered from band ' $b$ ' (-DHB) were comparable to peptides recovered from band 'e' (+DHB) (57 peptides corresponding to EntB, representing 78\% sequence coverage). Consistent with our analysis of band ' $\mathrm{f}$ ', twelve peptides corresponding to EntB were also obtained from band 'c' (DHB), representing $30 \%$ sequence coverage.

\section{Discussion}

The E. coli enzyme EntE is an essential component of the enterobactin biosynthetic pathway. Recently this protein has been the focus of studies probing ligand binding specificity ${ }^{15,16}$ as well as identifying novel compounds that can act as tight-binding EntE inhibitors. ${ }^{22,23}$ From previously reported kinetic characterizations we know that the release of the product 2,3-DHB-AMP is slow and rate-determining. By isothermal titration calorimetry we determined a direct, unambiguous measurement of the equilibrium binding constant $\left(\mathrm{K}_{\mathrm{D}}\right)$ of 2,3-DHB binding to EntE $(7.3 \mu \mathrm{M})$. This value is approximately three-fold higher than the reported 2,3-DHB $\mathrm{K}_{\mathrm{m}}$ value $(2.7 \mu \mathrm{M}){ }^{17}$ Although an equilibrium binding constant has not been reported for DHB-AMP binding to EntE, it has been found to be a competitive inhibitor of the substrate, with a $\mathrm{K}_{\mathrm{i}}$ value of $85 \mathrm{nM} .^{22}$ It is clear from our data, in conjunction with these previous findings, that the binding affinity of EntE for the 2,3-DHB substrate is much lower than that for the 2,3-DHB-AMP product. High-affinity binding of the DHB-AMP product to EntE ensures its efficient channeling to the holo-EntB ArCP domain. That the affinity of binding of the 2,3-DHB substrate is lower than that of the product suggests that considerable dissociation of the substrate to the bulk phase might occur 
prior to its adenylation. However, subsequent binding of the ATP co-substrate with closure of the P-loop could prevent this dissociation.

Our fluorescence emission spectra of H6-EntE showed that in the absence of 2,3-DHB there is a single emission maximum at $330 \mathrm{~nm}$ upon excitation at $280 \mathrm{~nm}$. When we added 2,3DHB to H6-EntE, we observed a new and large emission peak at $440 \mathrm{~nm}$ upon excitation at 280 $\mathrm{nm}$, the wavelength of maximal tyrosine excitation. Based on our experimental observations coupled with insights from our EntE homology model, we conclude that this emission peak is due to fluorescence resonance energy transfer (FRET) between EntE intrinsic fluorophore donors and the bound DHB acceptor. In the absence of H6-EntE, we observe that 2,3-DHB emits maximally at $440 \mathrm{~nm}$ with a maximal excitation wavelength of $306 \mathrm{~nm}$. Thus there is a spectral overlap between EntE intrinsic tyrosine fluorescence emission $\left(\lambda_{\mathrm{em}}=305 \mathrm{~nm}\right)$ and our observed DHB excitation maximum. Our fluorescence excitation spectra did not reveal a significant shift in the DHB excitation maxima when the substrate is bound to EntE (there is a slight $3 \mathrm{~nm}$ red shift in DHB $\lambda_{\text {ex }}$ to $309 \mathrm{~nm}$ ) indicating that observed fluorescence at $440 \mathrm{~nm}$ is not due to an alteration in the fluorescence properties of 2,3-DHB itself when bound to the EntE active site. When we collected spectra at an excitation wavelength of $275 \mathrm{~nm}$ instead of $280 \mathrm{~nm}$, we observed an increase in the $440 \mathrm{~nm}$ peak, not a decrease. This suggests that the FRET signal we observe is mostly due to excitation by instrinsic tyrosine donors proximal to the EntE active site, although we cannot rule out some contribution to the FRET signal by more distal tryptophan donors. Regardless of the exact nature of the EntE FRET donor residues, it is clear that the increase in observed fluorescence emission at $440 \mathrm{~nm}$ is due to 2,3-DHB binding to $H 6$-EntE. This increase is saturable with increasing DHB concentrations and follows hyperbolic single-site binding behavior that allowed us to obtain an $\mathrm{EC}_{50}$ value of 2,3-DHB binding to $H 6$-EntE that 
lowed us to obtain an $\mathrm{EC}_{50}$ value of 2,3-DHB binding to H6-EntE that agrees with our ITC outcomes.

Until now, our knowledge of the exact residues involved in EntE substrate binding has been limited. According to our EntE homology model, 2,3-DHB binds in an active site cavity proximal to five tyrosine residues. The closest of these residues is EntE Y236, which appears to be able to form a stacking interaction with the aromatic moiety of DHB. In the DhbE structure, Y236 does indeed stack with bound DHB. In our EntE model the Y236 aromatic ring is orthogonal to the DHB aromatic moiety, although the model indicates that the tyrosine side-chain has sufficient rotational degrees of freedom to facilitate stacking. In addition to Y236, our homology model reveals additional EntE residues that we predict to be involved in DHB binding. In the DhbE structure, residues H234 and K517 stabilize the carboxylate moiety of the substrate. ${ }^{20}$ In our model, the side chains of EntE residues H233 and K519 superimpose with these DhbE carboxylate-binding residues, predicting their role in substrate binding in the EntE active site. In addition, our model predicts that EntE residues N234 and S239 bind the 2- and 3-hydroxyl groups of 2,3-DHB. Here it is worth noting that our ITC data show that binding of 2,3-DHB to EntE has a negative entropy component. Binding of 2,3-DHB to EntE is therefore enthalpy driven, and not primarily due to hydrophobic stacking interactions with Y236. This is supported by our fluorescence studies that clearly show that EntE specifically binds 2,3-DHB. Neither 2,5DHB nor 3,5-DHB upon mixture with H6-EntE cause a quenching of intrinsic $H 6$-EntE fluorescence, nor do these isomers generate a FRET signal at $440 \mathrm{~nm}$ upon excitation at $280 \mathrm{~nm}$. The selectivity of H6-EntE binding to 2,3-DHB can be explained by the side-chain positions of the hydrogen bond donors N234 and S239 relative to the bound substrate as predicted by our model. In contrast, C5 of the bound 2,3-DHB is proximal to a loop containing EntE residues V306, 
G307, G308. None of these residues could stabilize a DHB isomer containing a hydroxyl group at position C5. Based on our model, in conjunction with our experimental data, we propose that EntE residues N234 and S239 are essential for determining EntE substrate-binding specificity.

Two recent studies have suggested that misacylation of EntB may occur due to the ability of EntE to adenylate non-physiological DHB isomers. In the one study, a $\sim 30 \%$ reduction in in vitro enterobactin biosynthesis was observed when 1,500 $\mu \mathrm{M}$ 2,4-DHB and 3,4-DHB were mixed with 2,3-DHB in a cell-free assay, showing that misacylation interfered with downstream NRPS processes. ${ }^{15}$ In the other study, it was found that holo-EntB could be acylated with 2,4DHB and 3,4-DHB when in the presence of EntE and $500 \mu \mathrm{M}$ of either DHB isomer. ${ }^{16}$ In these experiments, the concentrations of DHB isomers used was far in excess of the $\mathrm{K}_{\mathrm{D}}$ of 2,3-DHB binding to EntE as determined by ITC in this study. Our fluorescence experiments do not indicate any binding of 2,5-DHB or 3,5-DHB to EntE at what is likely to be a more physiologically relevant concentration $(50 \mu \mathrm{M})$, although the possibility remains that binding of these isomers may have occurred in a manner such that a FRET signal was not generated. While H6-EntE is selective for 2,3-DHB binding under our experimental conditions, we found that all DHB isomers examined (2,3-DHB, 2,5-DHB, and 3,5-DHB) were able to quench $H 6$-EntB intrinsic fluorescence to some degree. It is significant that 2,3-DHB was found to quench H6-EntB intrinsic fluorescence most efficiently, suggesting a binding preference for this isomer. Our farUV and near-UV circular dichroism data also established that 2,3-DHB can bind to H6-EntB, resulting in conformational changes to the enzyme. These results were unexpected, since 2,3DHB is not a substrate for the ICL domain of EntB nor the ArCP domain. Based on our results, we propose that EntB may possess an allosteric site that binds 2,3-DHB to regulate 2,3-DHB synthesis by feedback inhibition. It is reasonable that EntB would be a target for such regulation, 
since EntC, the first enzyme in the pathway, has been shown to be highly reversible. ${ }^{24}$ Given its reversibility, it is unlikely that the conversion of chorismate to isochorismate is the committed step of enterobactin biosynthesis, which would make the EntB-catalyzed conversion of isochorismate to 2,3-dihydro-dihydroxybenzoate the likely first committed step of the pathway. Our data suggest that not only could the DHB module be feedback-attenuated in the presence of excess intracellular 2,3-DHB binding to EntB, but also that interaction with EntE would be promoted through ligand-induced conformational changes in EntB, thus enhancing NRPS and enterobactin formation further decreasing intracelluar 2,3-DHB levels. We are now further investigating the nature of 2,3-DHB binding to EntB to address this hypothesis.

We used the FRET signal generated by 2,3-DHB binding to the EntE active site as a tool to measure interaction of $H 6$-EntE with $H 6$-EntB. We found that addition of $H 6$-EntB to a solution of H6-EntE saturated with 2,3-DHB resulted in significant quenching of the observed 2,3DHB-EntE FRET signal. We conclude that this quenching reflects a conformational change in the EntE active site upon interaction with EntB, resulting in alterations to the geometry and distance of the bound 2,3-DHB substrate relative to intrinsic EntE FRET donor residues and a subsequent decrease in the FRET signal. We observed that upon increasing EntB concentration, the 2,3-DHB-EntE FRET signal decreases until apparent saturation is achieved. Saturation of the quenching effect cannot be simply due to sequestration of 2,3-DHB as a result of its binding to H6-EntB, since the concentration of 2,3-DHB $(50 \mu \mathrm{M})$ was far greater than the concentration of H6-EntB $(3 \mu \mathrm{M})$ used in this assay. Our fluorescence data describing the EntE-EntB interaction fit to a sigmoidal curve, suggesting positive binding cooperativity. The EntB concentration at which quenching of the FRET signal is half-maximal gives us an $\mathrm{EC}_{50}$ value of the interaction affinity, which is in the low micromolar range, suggesting that EntE and EntB interact tran- 
siently. Since EntB must deliver acylated 2,3-DHB to EntF during NRPS, low-affinity proteinprotein interactions would allow EntB to interact in an asynchronous manner with both upstream and downstream partners in the NRPS assembly line. We also found that mixture of H6-EntB with H6-EntE in the presence of 2,3-DHB resulted in additional changes in near-UV CD signal intensities at wavelengths corresponding to contributions from aromatic chromophores in each protein. Such changes in the near-UV CD signal is generally due to alterations in the local environment of aromatic amino acids contributing to the signal, with reductions in peak intensities due to transitions to a more symmetric environment. We therefore conclude that our observed changes in the near-UV CD signals of H6-EntE and H6-EntB upon their mixture in the presence of 2,3-DHB are due to conformational rearrangements upon formation of the EntE-EntB interaction interface. These rearrangements may include conformational alterations of surface-exposed aromatic residues at the interface upon entering a more symmetric and ordered environment, and/or gross structural rearrangements in the EntB protein upon interaction with EntE.

Our pull-down assays establish that the EntB-EntE interaction can occur in situ between H6-EntB and chromosomally-expressed E. coli EntE. Furthermore, the presence of exogenously added 2,3-DHB greatly enhances the efficiency of EntE pulldown by H6-EntB. This is consistent with our $\mathrm{CD}$ data, which show gross EntB conformational rearrangements upon mixture with EntE only when 2,3-DHB is present. The binding of 2,3-DHB to EntE appears to subtly alter the surface of EntE in such a way that an EntB-EntE interaction interface is optimized. The conformational rearrangements in EntB observed by circular dichroism at both secondary- and tertiarystructure levels occur as a consequence of its interaction with substrate-loaded EntE. Our pulldown data show that binding of chromosomally-expressed EntE to H6-EntB bait is greatly en- 
hanced in the presence of exogenously added $(100 \mu \mathrm{M})$ 2,3-DHB, confirming that efficient formation of the EntE-EntB complex depends on ligand-loaded EntE and EntB.

That efficient pull-down did not occur in the absence of exogenously added 2,3-DHB strengthens our argument that the pool of free intracellular 2,3-DHB is normally low, presumably due to its direct channeling through the enterobactin biosynthetic machinery. Some further insights into EntB conformational flexibility may be obtained from our pull-down data. On our SDS-polyacrylamide gel of pull-down products, we observed bands at approximately $25 \mathrm{kDa}$ (Fig. 8, bands ' $c$ ' and ' $\mathrm{f}$ ') containing EntB peptides. We conclude that this is the result of EntB proteolysis. The ArCP domain of EntB resides between residues 214 - 285. The most C-terminal EntB peptide that we observed by mass spectrometry comprises residues $200-215$ (Table 1, row 41). Although only five peptides were recovered from the $25-\mathrm{kDa}$ band obtained in the presence of exogenous 2,3-DHB, we also recovered 12 peptides from the 25-kDa band pulled down in the absence of DHB (Fig. 8, band 'c', -DHB). The most C-terminal EntB peptide from this dataset also corresponded to residues $200-215$. Band ' $c$ ' therefore corresponds to the ICL domain of EntB, with proteolysis likely occurring in the linker region between the EntB ICL and ArCP domains. This suggests that in solution the interdomain region of EntB is solvent-exposed and flexible, and may thus play a role in EntB conformational rearrangements. It is also worth noting that we have measured EntE-EntB interaction in the absence of ATP, implying that ATP can still access the EntE active site subsequent to formation of the EntE-EntB complex. This opens up some intriguing lines of inquiry regarding the order of substrate binding and protein interaction.

In this study we have shown that binding of 2,3-DHB to the E. coli enterobactin biosynthetic enzyme EntE can be measured in a rapid and sensitive manner using fluorimetry. Observed changes in intrinsic EntE fluorescence upon binding of DHB can be clearly explained by 
the spatial distribution of tyrosine and tryptophan residues predicted by our homology model of the EntE structure. EntE and homologous AMP ligases from pathogenic bacteria are becoming targets for the development of novel synthetic inhibitors that rely on the high affinity of binding of DHB-AMP product. ${ }^{22,23}$ Fluorescence-based assays of the binding of DHB-like analogues, or of the inhibition of DHB binding in the case of non-fluorescent inhibitors could result in a more high-throughput approach to the development of such drugs. The fluorescence phenomenon that we have reported here also allows us to use DHB as a reporter of the EntE active site environment. Most importantly, we used this fluorescence phenomenon to measure the interaction of EntE with its immediate downstream enzymatic partner EntB. Taken together, our data show that EntE only interacts efficiently with EntB in the presence of 2,3-DHB. This transient complex is formed presumably to facilitate channeling of 2,3-DHB-AMP. Our results lead us to hypothesize that the enterobactin biosynthetic machinery has evolved to minimize free intracellular 2,3-DHB, which may be toxic to the bacterium (e.g., by inhibiting chorismate-dependent cellular processes such as aromatic amino acid biosynthesis), or which may inhibit other aspects of enterobactin biosynthesis and secretion (e.g., competing with enterobactin for binding to recognition sites on the efflux transporter EntS). We are now conducting further studies to expand our understanding of the EntB-EntE interaction in the larger context of molecular mechanisms inherent in the enterobactin biosynthetic assembly line.

\section{Materials and Methods}

Reagents. Unless otherwise noted, all reagents were purchased from BioShop Canada, Inc. (Burlington, Ontario). 2,3-DHB, 2,5-DHB and 3,5-DHB were purchased from Sigma-Aldrich. 
Homology Modeling of EntE. A homology-based model of EntE was obtained using the crystal structure of B. subtilis holo-DhbE (with bound 2,3-DHB and AMP) as a template (PDB code: 1md9)..$^{20}$ A structure-based alignment of the EntE amino acid sequence to that of DhbE was obtained using the FFAS03 server (http://ffas.ljcrf.edu/ffas-cgi/cgi/ffas.pl). ${ }^{25}$ This alignment was used as input for the modeling software Modeller 9v3 ${ }^{26}$ Structural superposition of the EntE homology model to the DhbE template structure was performed using $\mathrm{LSQMAN}^{27}$ in order to determine RMSD values. Model quality was assessed using the VADAR suite (http://redpoll.pharmacy.ualberta.ca/vadar/). ${ }^{21}$

Protein Expression and Purification. Escherichia coli AG-1 (endA1 recAl gyrA96 thi-1 relA1 glnV44 hsdR17(rK $\left.\left.m K^{+}\right)\right)$cells harboring pCA24N plasmids containing the genes encoding EntE and EntB with in-frame N-terminal hexahistidine tags (i.e., pCA24N-EntE and pCA24NEntB) were obtained from the ASKA repository..$^{28}$ The strains were grown at $37^{\circ} \mathrm{C}$ in $\mathrm{LB}$ broth containing $30 \mu \mathrm{g} / \mathrm{ml}$ of chloramphenicol to a final $\mathrm{OD}_{600}$ between $0.5-0.6$. Overexpression of hexahistidine-tagged EntE or hexahistidine-tagged EntB (henceforth referred to as H6-EntE and H6-EntB, respectively) was induced in log-phase cells with the addition of IPTG to a concentration of $1 \mathrm{mM}$. After an additional 3-hour growth at $37^{\circ} \mathrm{C}$, cells were harvested by centrifugation (15 min at 5,000 g). Pellets were resuspended in BugBuster Master Mix (Novagen) and incubated for $30 \mathrm{~min}$ at room temperature with constant agitation. Cell lysates were centrifuged (50 min at 10,000 g) and supernatants were recovered. Clarified lysates containing H6-EntE or H6EntB were loaded onto 10-ml Profinity IMAC columns (Bio-Rad Laboratories) connected to a BioLogic DuoFlow FPLC system (Bio-Rad Laboratories). Fractions containing washed H6-EntE or H6-EntB were eluted by linear imidazole gradients (10 - $500 \mathrm{mM}$ imidazole) in a buffer containing $50 \mathrm{mM}$ Hepes (pH 8.0), $100 \mathrm{mM} \mathrm{KCl}$, and $1.0 \mathrm{mM}$ TCEP. We determined that IMAC 
purification yielded near-homogenous (> 95\% purity) H6-EntE. However, H6-EntB required additional purification on a 1-ml UNOsphere Q anion exchange column (Bio-Rad Laboratories) to achieve a near-homogeneous ( $>95 \%$ purity) H6-EntB fraction. Pooled fractions containing purified $H 6$-EntE and $H 6$-EntB were dialyzed against $50 \mathrm{mM}$ Hepes (pH 8.0), $100 \mathrm{mM} \mathrm{KCl}, 1.0 \mathrm{mM}$ TCEP, and $15 \%$ glycerol. Dialyzed samples were stored at $-20{ }^{\circ} \mathrm{C}$. We used the Enzchek pyrophosphate release assay (Invitrogen) to measure H6-EntE steady-state enzyme activity and found its specific activity to be comparable to that reported earlier for non-hexahistidine-tagged EntE. ${ }^{17}$ Stability of H6-EntE was monitored by enzymatic assays, and no loss of activity was observed after extended ( $>14$ days) storage at $4{ }^{\circ} \mathrm{C}$ or after long ( $>4$ weeks) periods of storage at $-20{ }^{\circ} \mathrm{C}$ followed by subsequent thawing.

Isothermal Titration Calorimetry. Heat generated by the binding of 2,3-DHB to H6-EntE was measured using a VP-ITC isothermal titration microcalorimeter (cell volume $=1.36 \mathrm{ml}$; Microcal, Inc.). 2,3-DHB dissolved to a concentration of $500 \mu \mathrm{M}$ in Buffer A (50 mM Hepes (pH 8.0), $100 \mathrm{mM} \mathrm{KCl}, 1 \mathrm{mM}$ TCEP, and 15\% glycerol) was injected into a cell containing $50 \mu \mathrm{M}$ purified H6-EntE dialyzed against Buffer A. Protein concentration was determined by absorption at $280 \mathrm{~nm}$ as measured on a Thermo Genesys 10 spectrophotometer and using a molar extinction coefficient predicted from the H6-EntE primary amino acid sequence $\left(57,800 \mathrm{M}^{-1} \mathrm{~cm}^{-1}\right)$. Data from 24 injections (injection volume $=10 \mu$ l) were collected at $293 \mathrm{~K}$ while the contents of the cell were stirred at $300 \mathrm{rpm}$. An initial $2-\mu 1$ injection was not included in the data analysis. A spacing time of 240 seconds occurred between each injection. Heats of injection were corrected by subtraction of heats of dilution generated by injecting $500 \mu \mathrm{M} 2,3$-DHB into the sample cell containing Buffer A. Calorimetry data were analyzed using Origin 5.0 (Microcal, Inc.) by fitting 
the corrected heats of injection to an equation describing single-site ligand binding. Values of $\Delta \mathrm{H}$ (in kcal mol${ }^{-1}$ ), $\mathrm{S}$ (in cal $\mathrm{mol}^{-1} \mathrm{~K}^{-1}$ ) and $\mathrm{K}_{\mathrm{D}}$ were obtained from the Origin software.

Fluorescence Spectroscopy. Fluorescence emission and excitation spectra of purified H6-EntE and H6-EntB in the presence and absence of 2,3-DHB, 2,5-DHB, or 3,5-DHB were collected at room temperature using a Shimadzu RF-5301PC spectrofluorometer. For emission spectra, the excitation wavelength was fixed at $275 \mathrm{~nm}, 280 \mathrm{~nm}$, or $295 \mathrm{~nm}$ and fluorescence emission intensities were recorded from $300 \mathrm{~nm}$ to $550 \mathrm{~nm}$. For excitation spectra, the emission wavelength was fixed at $440 \mathrm{~nm}$ and fluorescence intensities were collected upon changing the excitation wavelength from $200 \mathrm{~nm}$ to $350 \mathrm{~nm}$. Excitation and emission slits were set to $3 \mathrm{~nm}$. Measurements were performed using a $0.5 \mathrm{ml}$ quartz cuvette (10 mm path length) containing purified H6EntE or H6-EntB diluted to a final concentration of $3 \mu \mathrm{M}$ in Buffer A. Each reported fluorescence spectrum represents the average of three scans $(2 \mathrm{~nm} / \mathrm{sec})$. Fluorescence intensities were corrected for inner filter effects according to the equation: $\mathrm{F}_{\mathrm{corr}}=\mathrm{F}_{\mathrm{obs}} \times 10^{((\mathrm{ODex}+\mathrm{ODem}) / 2)}$, where $\mathrm{F}_{\mathrm{obs}}$ is the observed fluorescence intensity, $\mathrm{OD}_{\mathrm{ex}}$ is the optical density of the solution at the excitation wavelength, $\mathrm{OD}_{\mathrm{em}}$ is the optical density of the solution at the emission wavelength. Fluorescence emission spectra of H6-EntE were obtained with varying concentrations of 2,3-DHB (5, $10,20,30,40,50,60,70$, and $80 \mu \mathrm{M})$. Half-maximal effective concentration $\left(\mathrm{EC}_{50}\right)$ values describing 2,3-DHB binding to H6-EntE were calculated from fluorescence emission data (either intensity increase at $440 \mathrm{~nm}$ or decrease at $330 \mathrm{~nm}$ ) using the computer program Origin 5.0 and fitting the data to a hyperbolic equation to determine $\mathrm{EC}_{50}$ values. The EntE-EntB interaction was measured by fluorescence using a solution of H6-EntE $(2.5 \mu \mathrm{M})$ in Buffer A plus $50 \mu \mathrm{M}$ 2,3-DHB to which increasing concentrations of H6-EntB (0.4- 3.4 $\mu \mathrm{M})$ were added. 
In this study, the fluorescence spectra of all protein solutions mixed with a given concentration of 2,3-DHB, 2,5-DHB, or 3,5-DHB were corrected by subtracting fluorescence emission spectra of the appropriate DHB isomer collected at the same concentration in the absence of protein. Furthermore, all corrections for dilution factors were applied when appropriate.

Circular Dichroism. Circular dichroism experiments were performed on a Jasco J-815 CD spectrophotometer connected to a temperature-controlled circulating water bath. Far-UV spectra of H6-EntE $(7.5 \mu \mathrm{M})$ and $H 6$-EntB $(2.5 \mu \mathrm{M})$ solutions were collected at $20^{\circ} \mathrm{C}$ between $200 \mathrm{~nm}$ and $260 \mathrm{~nm}(1 \mathrm{~nm}$ bandwidth $)$ in $0.2-\mathrm{nm}$ steps at a rate of $100 \mathrm{~nm} / \mathrm{min}(0.25 \mathrm{sec}$ response) using a cell with a path length of $0.2 \mathrm{~cm}$. Each far-UV spectrum presented here is an average of five scans. Near-UV spectra of $H 6$-EntE $(25 \mu \mathrm{M})$ and $H 6$-EntB $(15.6 \mu \mathrm{M})$ were collected at $20^{\circ} \mathrm{C}$ between $250 \mathrm{~nm}$ and $320 \mathrm{~nm}(1 \mathrm{~nm}$ bandwidth) in $0.2-\mathrm{nm}$ steps at a rate of $20 \mathrm{~nm} / \mathrm{min}(2 \mathrm{sec}$ response) using a cell with a path length of $2.0 \mathrm{~cm}$. Each near-UV spectrum presented here is an average of two scans. Both near-UV and far-UV spectra were collected from solutions of individual proteins and of mixtures of $H 6$-EntE and $H 6$-EntB. In all cases, protein solutions for CD analysis were dialyzed against $25 \mathrm{mM}$ Tris (pH 8.0), $1 \mathrm{mM} \mathrm{TCEP,} 100 \mathrm{mM} \mathrm{KCl}$, and 15\% glycerol and then diluted to the desired concentration in the same buffer. Spectra were collected in the presence and absence of $50 \mu \mathrm{M} 2,3$-DHB. Spectra collected in the presence of $50 \mu \mathrm{M} 2,3-$ DHB were corrected by subtraction of signal contribution from $50 \mu \mathrm{M} 2,3$-DHB dissolved in the same buffer in the absence of protein.

Pull-down Assays. Pull-down assays were performed using the ProFound Pull-Down PolyHis Protein:Protein Interaction Kit (Pierce, Rockford, IL). Cobalt chelate columns were equilibrated with washing solution (1:1 mixture of Tris Buffered Saline ( $25 \mathrm{mM}$ Tris- $\mathrm{HCl}(\mathrm{pH} 7.2), 150 \mathrm{mM}$ $\mathrm{NaCl}$ ) with ProFound Lysis Buffer, and imidazole to a concentration of $40 \mathrm{mM}$ ). H6-EntB (130 
$\mu \mathrm{g}$ ), the bait protein, was immobilized to cobalt chelate columns by incubation for 1 hour at $4{ }^{\circ} \mathrm{C}$ with gentle shaking. Cell lysates containing prey proteins were prepared from E. coli BW25113 cells grown for $16 \mathrm{~h}$ at $37^{\circ} \mathrm{C}$ in iron-free M63 minimal media in the presence of $75 \mu \mathrm{M} 2,2^{\prime}$ dipyridyl. Lysates were added to the cobalt columns in the presence or absence of $100 \mu \mathrm{M} 2,3-$ DHB and then incubated at $4{ }^{\circ} \mathrm{C}$ with gentle shaking for 2 hours. The columns were washed 8 times with washing solution. Bait-prey protein complexes were eluted by the addition of imidazole to a final concentration of $300 \mathrm{mM}$. Eluted proteins were separated on $10 \% \mathrm{SDS}-$ polyacrylamide gels. After electrophoresis, gels were stained using the SilverQuest Silver Staining kit (Invitrogen). Bands of interest were excised from the gels and proteins were subjected to in-gel tryptic digestion. Recovered samples were subjected to LC-MS/MS analysis using Bruker ESI Ion Trap and Agilent LC 1100 mass spectrometers at the McGill Mass Spectrometry Core Facility (McGill University, Montreal). Briefly, samples obtained from gel slices were dried and then resuspended in $97 \%$ acetonitrile ( $\mathrm{ACN}) / 0.2 \%$ formic acid. Resuspended samples were injected in the mass spectrometer, and a gradient of $0-70 \%$ ( $95 \%$ water $/ 0.5 \% \mathrm{ACN}$ to $9.8 \%$ water/90\%ACN $/ 0.2 \%$ formic acid) was used to elute the peptides. The resulting data were searched against the NCBI database using MASCOT (Matrix Science). 


\section{Acknowledgements}

We thank Drs. Eric Massé, Jack Kornblatt, Judith Kornblatt, and Joanne Turnbull for their helpful comments and discussions related to this manuscript. We also thank Dr. Jack Kornblatt for his kind assistance with our ITC experiments, and Dr. Michael Mourez for his helpful suggestions regarding our fluorescence experiments. We also acknowledge the McGill Mass Spectrometry Core Facility for their assistance in performing mass spectrometry experiments and analysis of resulting data. 


\section{References}

1. Wandersman C. \& Delepelaire P. (2004). Bacterial iron sources: from siderophores to hemophores. Anпu Rev Microbiol 58, 611-647.

2. Miethke M. \& Marahiel M. A. (2007). Siderophore-based iron acquisition and pathogen control. Microbiol Mol Biol Rev 71, 413-451.

3. Raymond K. N., Dertz E. A. \& Kim S. S. (2003). Enterobactin: an archetype for microbial iron transport. Proc Natl Acad Sci U S A 100, 3584-3588.

4. Ma L., Kaserer W., Annamalai R., Scott D. C., Jin B., Jiang X. X., Xiao Q. B., Maymani H., Massis L. M., Ferreira L. C. S., Newton S. M. C. \& Klebba P. E. (2007). Evidence of ball-andchain transport of ferric enterobactin through FepA. J Biol Chem 282, 397-406.

5. Fischbach M. A., Lin H., Liu D. R. \& Walsh C. T. (2006). How pathogenic bacteria evade mammalian sabotage in the battle for iron. Nat Chem Biol 2, 132-138.

6. Fischbach M. A., Lin H., Zhou L., Yu Y., Abergel R. J., Liu D. R., Raymond K. N., Wanner B. L., Strong R. K., Walsh C. T., Aderem A. \& Smith K. D. (2006). The pathogen-associated iroA gene cluster mediates bacterial evasion of lipocalin 2. Proc Natl Acad Sci U S A 103, $16502-16507$.

7. Liu J., Duncan K. \& Walsh C. T. (1989). Nucleotide sequence of a cluster of Escherichia coli enterobactin biosynthesis genes: identification of entA and purification of its product 2,3dihydro-2,3-dihydroxybenzoate dehydrogenase. J Bacteriol 171, 791-798.

8. Nahlik M. S., Fleming T. P. \& McIntosh M. A. (1987). Cluster of genes controlling synthesis and activation of 2,3-dihydroxybenzoic acid in production of enterobactin in Escherichia coli. $J$ Bacteriol 169, 4163-4170. 
9. Nahlik M. S., Brickman T. J., Ozenberger B. A. \& McIntosh M. A. (1989). Nucleotide sequence and transcriptional organization of the Escherichia coli enterobactin biosynthesis cistrons entB and entA. J Bacteriol 171, 784-790.

10. Ozenberger B. A., Brickman T. J. \& McIntosh M. A. (1989). Nucleotide sequence of Escherichia coli isochorismate synthetase gene entC and evolutionary relationship of isochorismate synthetase and other chorismate-utilizing enzymes. J Bacteriol 171, 775-783.

11. Woodrow G. C., Young I. G. \& Gibson F. (1975). Mu-induced polarity in the Escherichia coli K-12 ent gene cluster: evidence for a gene (entG) involved in the biosynthesis of enterochelin. J Bacteriol 124, 1-6.

12. Gehring A. M., Mori I. \& Walsh C. T. (1998). Reconstitution and characterization of the $E s-$ cherichia coli enterobactin synthetase from EntB, EntE, and EntF. Biochemistry 37, 2648-2659.

13. Lambalot R. H., Gehring A. M., Flugel R. S., Zuber P., LaCelle M., Marahiel M. A., Reid R., Khosla C. \& Walsh C. T. (1996). A new enzyme superfamily - the phosphopantetheinyl transferases. Chem Biol 3, 923-936.

14. Leduc D., Battesti A. \& Bouveret E. (2007). The Hotdog thioesterase EntH (YbdB) plays a role in vivo in optimal enterobactin biosynthesis by interacting with the ArCP domain of EntB. $J$ Bacteriol 189, 7112-7126.

15. Guo Z. F., Sun Y., Zheng S. \& Guo Z. (2009). Preferential hydrolysis of aberrant intermediates by the type II thioesterase in Escherichia coli nonribosomal enterobactin synthesis: substrate specificities and mutagenic studies on the active-site Residues. Biochemistry .

16. Chen D., Wu R., Bryan T. L. \& Dunaway-Mariano D. (2009). In vitro kinetic analysis of substrate specificity in enterobactin biosynthetic lower pathway enzymes provides insight into the biochemical function of the hot dog-fold thioesterase EntH. Biochemistry 48, 511-513. 
17. Rusnak F., Faraci W. S. \& Walsh C. T. (1989). Subcloning, expression, and purification of the enterobactin biosynthetic enzyme 2,3-dihydroxybenzoate-AMP ligase: demonstration of enzyme-bound (2,3-dihydroxybenzoyl)adenylate product. Biochemistry 28, 6827-6835.

18. Ehmann D. E., Shaw-Reid C. A., Losey H. C. \& Walsh C. T. (2000). The EntF and EntE adenylation domains of Escherichia coli enterobactin synthetase: sequestration and selectivity in acyl-AMP transfers to thiolation domain cosubstrates. Proc Natl Acad Sci U S A 97, 2509-2514.

19. Drake E. J., Nicolai D. A. \& Gulick A. M. (2006). Structure of the EntB multidomain nonribosomal peptide synthetase and functional analysis of its interaction with the EntE adenylation domain. Chem Biol 13, 409-419.

20. May J. J., Kessler N., Marahiel M. A. \& Stubbs M. T. (2002). Crystal structure of DhbE, an archetype for aryl acid activating domains of modular nonribosomal peptide synthetases. Proc Natl Acad Sci U S A 99, 12120-12125.

21. Willard L., Ranjan A., Zhang H., Monzavi H., Boyko R. F., Sykes B. D. \& Wishart D. S. (2003). VADAR: a web server for quantitative evaluation of protein structure quality. Nucleic Acids Res 31, 3316-3319.

22. Callahan B. P., Lomino J. V. \& Wolfenden R. (2006). Nanomolar inhibition of the enterobactin biosynthesis enzyme, EntE: synthesis, substituent effects, and additivity. Bioorg Med Chem Lett 16, 3802-3805.

23. Miethke M., Bisseret P., Beckering C. L., Vignard D., Eustache J. \& Marahiel M. A. (2006). Inhibition of aryl acid adenylation domains involved in bacterial siderophore synthesis. FEBS $J$ 273, 409-419. 
24. Liu J., Quinn N., Berchtold G. A. \& Walsh C. T. (1990). Overexpression, purification, and characterization of isochorismate synthase (EntC), the first enzyme involved in the biosynthesis of enterobactin from chorismate. Biochemistry 29, 1417-1425.

25. Jaroszewski L., Rychlewski L., Li Z., Li W. \& Godzik A. (2005). FFAS03: a server for profile-profile sequence alignments. Nucleic Acids Res 33, W284-W288.

26. Sali A. \& Blundell T. L. (1993). Comparative protein modelling by satisfaction of spatial restraints. J Mol Biol 234, 779-815.

27. Kleywegt G. J. (1999). Experimental assessment of differences between related protein crystal structures. Acta Crystallogr D Biol Crystallogr 55, 1878-1884.

28. Kitagawa M., Ara T., Arifuzzaman M., Ioka-Nakamichi T., Inamoto E., Toyonaga H. \& Mori H. (2005). Complete set of ORF clones of Escherichia coli ASKA library (a complete set of E. coli $\mathrm{K}-12$ ORF archive): unique resources for biological research. DNA Res 12, 291-299 
Table 1. MASCOT analysis of peptides recovered from pull-down assay of irondepleted E. coli BW25113 lysates and identified by mass spectrometry.

\begin{tabular}{|c|c|c|c|c|c|}
\hline $\begin{array}{l}\text { Band on } \\
\text { Gel }^{a}\end{array}$ & $\mathrm{DHB}^{b}$ & Protein & $\begin{array}{c}\text { Residue } \\
\text { Range }\end{array}$ & $\begin{array}{l}\text { Delta Avg. } \\
(\mathrm{Da})^{c}\end{array}$ & Frequency $^{d}$ \\
\hline $\mathrm{d}$ & + & EntE & $8-14$ & 0.2490 & 2 \\
\hline d & + & EntE & $20-33$ & 0.2722 & 20 \\
\hline d & + & EntE & $34-47$ & 0.3206 & 7 \\
\hline d & + & EntE & $53-66$ & 0.2264 & 16 \\
\hline d & + & EntE & $96-109$ & 0.6179 & 8 \\
\hline d & + & EntE & $110-128$ & 0.3726 & 11 \\
\hline d & + & EntE & $129-150$ & 0.2852 & 1 \\
\hline d & + & EntE & $203-212$ & 0.1955 & 3 \\
\hline $\mathrm{d}$ & + & EntE & $213-225$ & 0.4819 & 1 \\
\hline d & + & EntE & $271-296$ & 0.2850 & 5 \\
\hline d & + & EntE & $312-343$ & 0.4175 & 1 \\
\hline d & + & EntE & $320-343$ & 0.4190 & 33 \\
\hline d & + & EntE & $351-382$ & 0.4925 & 2 \\
\hline d & + & EntE & $397-430$ & 0.4962 & 2 \\
\hline d & + & EntE & $442-453$ & 0.2739 & 39 \\
\hline d & + & EntE & $454-473$ & 0.2801 & 3 \\
\hline d & + & EntE & $474-481$ & 0.2641 & 1 \\
\hline d & + & EntE & $503-520$ & 0.4018 & 2 \\
\hline d & + & EntE & $507-520$ & 0.2502 & 5 \\
\hline $\mathrm{e}$ & + & EntB & $6-21$ & 0.4196 & 2 \\
\hline e & + & EntB & $22-30$ & 0.2724 & 5 \\
\hline e & + & EntB & $69-81$ & 0.4872 & 2 \\
\hline $\mathrm{e}$ & + & EntB & $89-101$ & 0.3311 & 15 \\
\hline $\mathrm{e}$ & + & EntB & $112-123$ & 0.3618 & 15 \\
\hline $\mathrm{e}$ & + & EntB & $112-125$ & 0.2588 & 1 \\
\hline e & + & EntB & $132-139$ & 0.2146 & 1 \\
\hline e & + & EntB & $144-167$ & 0.2988 & 3 \\
\hline $\mathrm{e}$ & + & EntB & $168-183$ & 0.2271 & 7 \\
\hline $\mathrm{e}$ & + & EntB & $200-215$ & 0.4174 & 17 \\
\hline e & + & EntB & $220-247$ & 0.4461 & 18 \\
\hline $\mathrm{e}$ & + & EntB & $220-254$ & 0.6332 & 1 \\
\hline e & + & EntB & $257-269$ & 0.2950 & 2 \\
\hline $\mathrm{e}$ & + & EntB & $257-269$ & 0.2950 & 2 \\
\hline e & + & EntB & $258-269$ & 0.2689 & 2 \\
\hline e & + & EntB & $270-278$ & 0.2102 & 3 \\
\hline $\mathrm{f}$ & + & EntB & $6-21$ & 0.2932 & 1 \\
\hline $\mathrm{f}$ & + & EntB & $22-30$ & 0.2004 & 1 \\
\hline $\mathrm{f}$ & + & EntB & $112-123$ & 0.2211 & 2 \\
\hline $\mathrm{f}$ & + & EntB & $200-215$ & 0.2270 & 1 \\
\hline
\end{tabular}

${ }^{a}$ see Figure $8 ;{ }^{b}$ exogenous 2,3-DHB added to lysate prior to pull-down; ${ }^{c} \mid$ (observed peptide mass $)$ - (expected peptide mass from sequence $) \mid{ }^{d}$ frequency of observed peptide recovered from gel slice. 


\section{Figure Legends}

Fig. 1. Scheme of reaction catalyzed by $E$. coli EntE.

Fig. 2. Homology-based model of $\boldsymbol{E}$. coli EntE. (a) Structure-based alignment of EntE to $B$. subtilis DhbE primary amino acid sequence. Positions of identity shown as white letters with black background. Positions of similarity shown as black letters on grey background. Residues are numbered according to mature primary amino acid sequences. (b) Structural superposition of EntE homology model to DhbE structure (PDB code: 1md9). EntE model shown as blue coil. DhbE structure shown as green coil. Position of DHB coordinates from DhbE structure shown as sticks and colored according to element. (c) Close-up view of DHB in the active sites of DhbE (green coil) and superimposed EntE (blue coil). EntE tyrosine residues within $10 \AA$ of DHB shown as blue sticks. (d) Close-up view of DHB in the active site of the EntE homology model (blue coil) showing EntE residues (white sticks, atoms colored according to element) predicted to form hydrogen bonds with 2,3-DHB substrate (yellow sticks, atoms colored according to element). Molecular graphics were generated using PyMOL (http://www.pymol.org).

\section{Fig. 3. Isothermal titration calorimetry of H6-EntE in the presence and absence of 2,3-}

DHB. Upper panel: Heats of injection of $500 \mu \mathrm{M}$ DHB into a cell containing $50 \mu \mathrm{M}$ H6-EntE. Lower panel: Data from upper panel integrated and plotted as a function of molar ratio of DHB after subtraction of heats generated by injection of $500 \mu \mathrm{M}$ DHB into buffer. Integrated heats shown as black squares. Single-site binding model shown as solid line.

Fig. 4. Fluorescence measurements 2,3-DHB binding to H6-EntE. (a) Fluorescence emission spectra $\left(\lambda_{\mathrm{ex}}=280 \mathrm{~nm}\right)$ of solutions containing: $3 \mu \mathrm{M}$ H6-EntE (black circles); $50 \mu \mathrm{M} 2,3-\mathrm{DHB}$ (magenta circles); $3 \mu \mathrm{M} H 6$-EntE and $50 \mu \mathrm{M}$ 2,3-DHB (green circles). (b) Fluorescence excita- 
tion spectra $\left(\lambda_{\mathrm{em}}=440 \mathrm{~nm}\right)$ of solutions containing: $50 \mu \mathrm{M} 2,3-\mathrm{DHB}$ (magenta circles); $50 \mu \mathrm{M}$ 2,3-DHB and $3 \mu \mathrm{M} H 6$-EntE (green circles). (c) Increase in fluorescence emission at $440 \mathrm{~nm}$ as a function of 2,3-DHB concentration. Fluorescence emission at $440 \mathrm{~nm}$ corrected by baseline fluorescence emission of $H 6$-EntE in the absence of 2,3-DHB, and by 2,3-DHB emission in the absence of H6-EntE (black squares). Fit of data to hyperbolic curve describing single-site binding (solid line). (d) Quenching of fluorescence emission at $330 \mathrm{~nm}$ as a function of 2,3-DHB concentration. Fluorescence quenching was calculated as $\left(\left(\mathrm{F}_{\mathrm{o}}-\mathrm{F}\right) / \mathrm{F}_{\mathrm{o}}\right) \times 100$, where $\mathrm{F}_{\mathrm{o}}$ is the initial fluorescence intensity observed at $330 \mathrm{~nm}$ in the absence of 2,3-DHB. Fluorescence quenching (black squares). Fit of data to hyperbolic curve describing single-site binding (solid line).

Fig. 5. Binding of DHB isomers to H6-EntE and H6-EntB. (a) Fluorescence emission spectra $\left(\lambda_{\mathrm{ex}}=280 \mathrm{~nm}\right)$ of solutions containing: $3 \mu \mathrm{M}$ H6-EntB (black circles); $3 \mu \mathrm{M}$ H6-EntB and 50 $\mu \mathrm{M}$ 2,3-DHB (green circles); $3 \mu \mathrm{M}$ H6-EntB and $50 \mu \mathrm{M}$ 2,5-DHB (red circles); $3 \mu \mathrm{M} H 6$-EntB and $50 \mu \mathrm{M} 3,5$-DHB (blue circles). (b) Fluorescence emission spectra ( $\lambda_{\mathrm{ex}}=280 \mathrm{~nm}$ ) of solutions containing: $3 \mu \mathrm{M}$ H6-EntE (black circles); $3 \mu \mathrm{M}$ H6-EntE and $50 \mu \mathrm{M}$ 2,3-DHB (green circles); 3 $\mu \mathrm{M}$ H6-EntE and $50 \mu \mathrm{M}$ 2,5-DHB (red circles); $3 \mu \mathrm{M}$ H6-EntE and $50 \mu \mathrm{M}$ 3,5-DHB (blue circles).

Fig. 6. Binding of H6-EntB to H6-EntE in the presence of 2,3-DHB. Percentage quenching (see Fig. 4, legend) of the $440 \mathrm{~nm}$ H6-EntE-2,3-DHB FRET signal (solid dots) as a function of H6-EntB concentration. Data were fit to a sigmoidal curve describing cooperative binding using SigmaPlot.

Fig. 7. Circular dichroism spectra of H6-EntE and H6-EntB in the presence and absence of DHB. (a) Far-UV circular dichroism spectra of protein solutions in the absence of 2,3-DHB : 
H6-EntE (blue circles); H6-EntB (red circles); mixture of $\{H 6$-EntE $+H 6$-EntB $\}$ with H6-EntB signal subtracted (cyan circles); mixture of $\{H 6$-EntE $+H 6$-EntB $\}$ with H6-EntE signal subtracted (magenta circles). (b) Far-UV circular dichroism spectra of protein solutions in the presence of $50 \mu \mathrm{M}$ 2,3-DHB : H6-EntE (blue circles); H6-EntB (red circles); mixture of $\{H 6$-EntE + H6EntB $\}$ with H6-EntB signal subtracted (cyan circles); mixture of $\{H 6$-EntE $+H 6$-EntB $\}$ with H6-EntE signal subtracted (magenta circles). (c) Near-UV circular dichroism spectra of protein solutions in the absence of 2,3-DHB : H6-EntE (blue circles); H6-EntB (red circles); mixture of $\{H 6-E n t E+H 6$-EntB $\}$ with H6-EntB signal subtracted (cyan circles); mixture of $\{H 6$-EntE $+H 6$ EntB $\}$ with H6-EntE signal subtracted (magenta circles). (d) Near-UV circular dichroism spectra of protein solutions in the presence of $50 \mu \mathrm{M}$ 2,3-DHB : H6-EntE (blue circles); H6-EntB (red circles); mixture of $\{H 6-\mathrm{EntE}+H 6-\mathrm{EntB}\}$ with $H 6$-EntB signal subtracted (cyan circles); mixture of $\{$ H6-EntE $+H 6$-EntB $\}$ with H6-EntE signal subtracted (magenta circles).

Fig. 8. Pull-down assay of proteins binding to H6-EntB bait. Silver-stained $10 \%$ SDSpolyacrylamide gel of proteins eluted from $\mathrm{Co}^{2+}$-chelate beads. Numbers to left of gel represent positions of bands of known molecular weight markers (not shown). Lane '-DHB': proteins isolated from beads exposed to lysate of iron-starved E. coli BW25338 with no exogenously added 2,3-DHB. Lane '+DHB': proteins isolated from beads exposed to lysate of iron-starved E. coli BW25338 cells with exogenously-added 2,3-DHB $(50 \mu \mathrm{M})$. The identity of proteins in bands 'b'-'f' were determined by LC-MS/MS analysis of bands excised from gel. Bands 'a' and 'd': $E$. coli EntE; Bands 'b' and 'e': E. coli EntB; Bands 'c' and 'f': E. coli EntB (proteolysed). 
Figure 1.

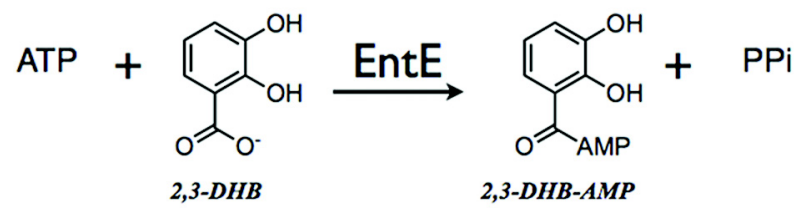


Figure 2.

A

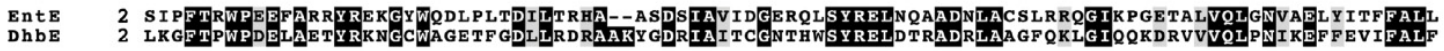

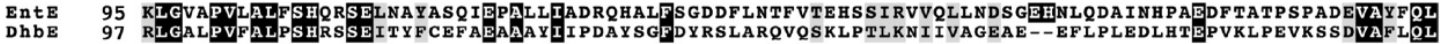

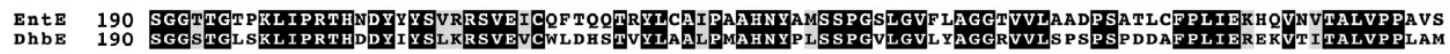

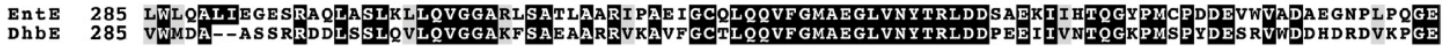

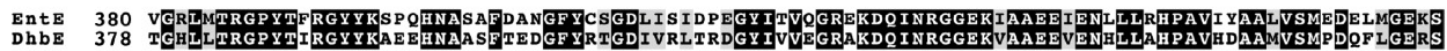

$\begin{array}{lll}\text { EntE } & 475 & \text { CAYLVVKEP-LRAVQVRRFLREGGIAEFKLPDRVECVDSLPLTAVGKVDKKOLROWLASRASA } \\ \text { DhbE } & 473 \text { CVFIIPRDEAPKAELKAFLRERGLAYKIPDRVEVESFOIGGKVSKALREAISEKLLA }\end{array}$

B

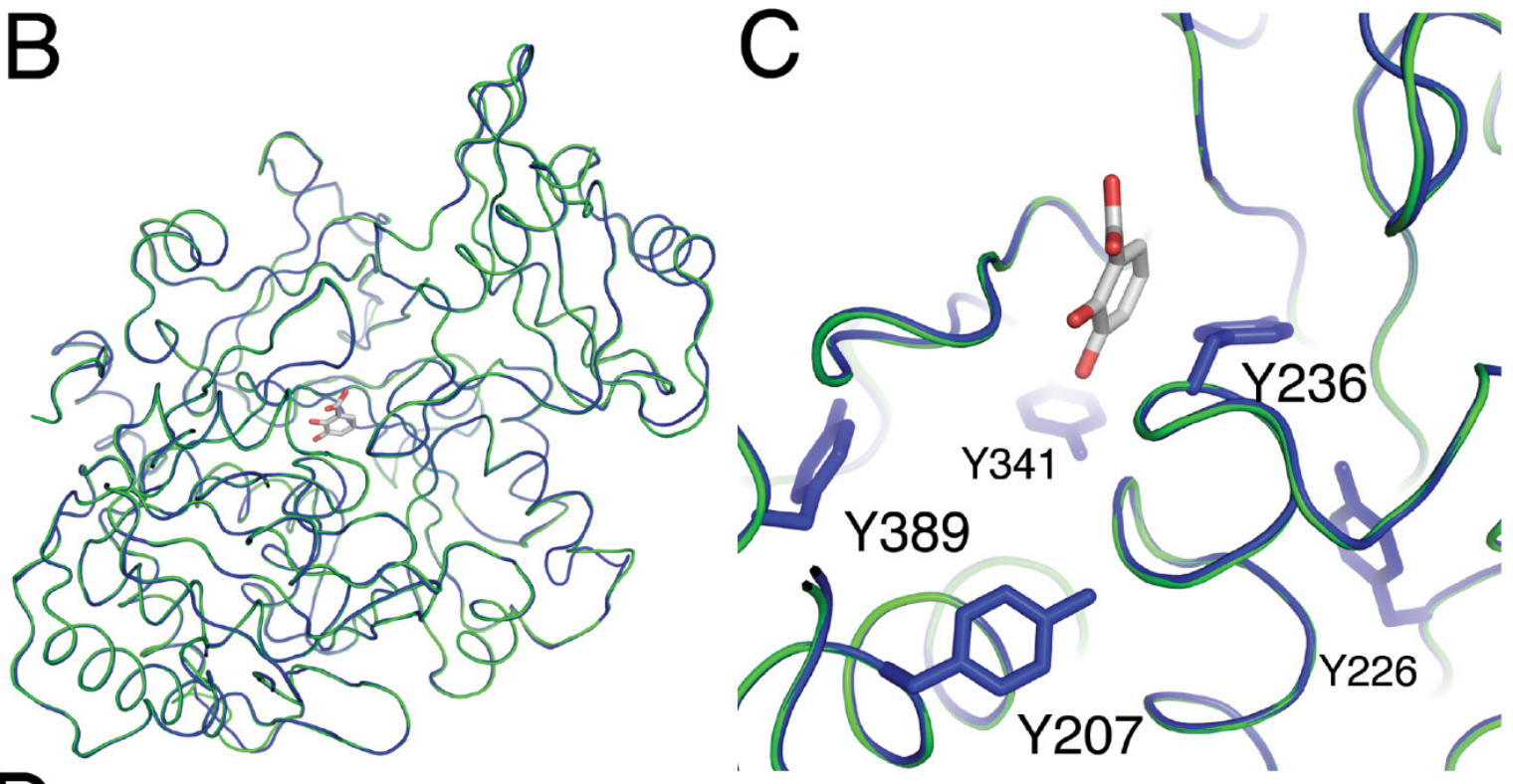

C

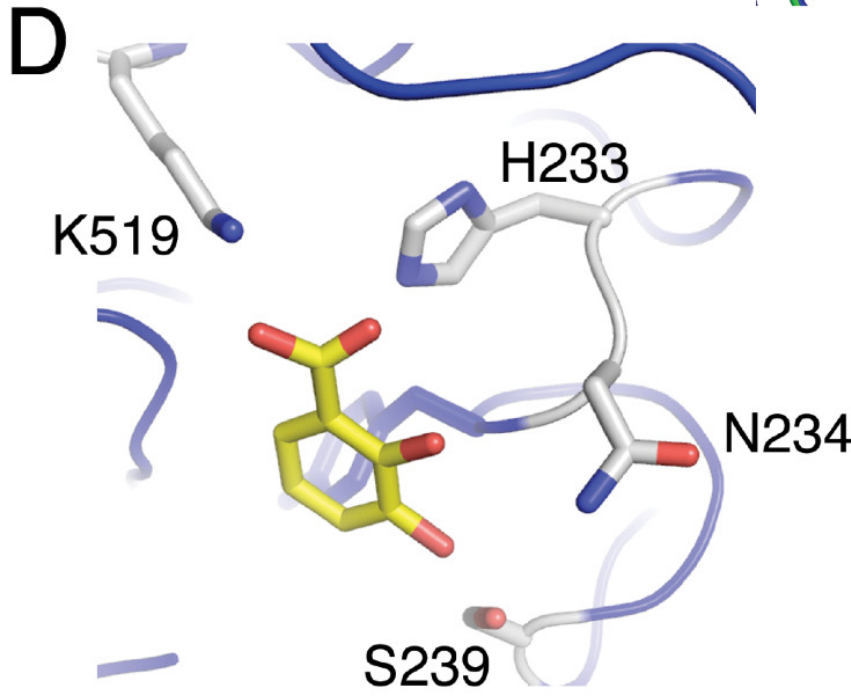


Figure 3.

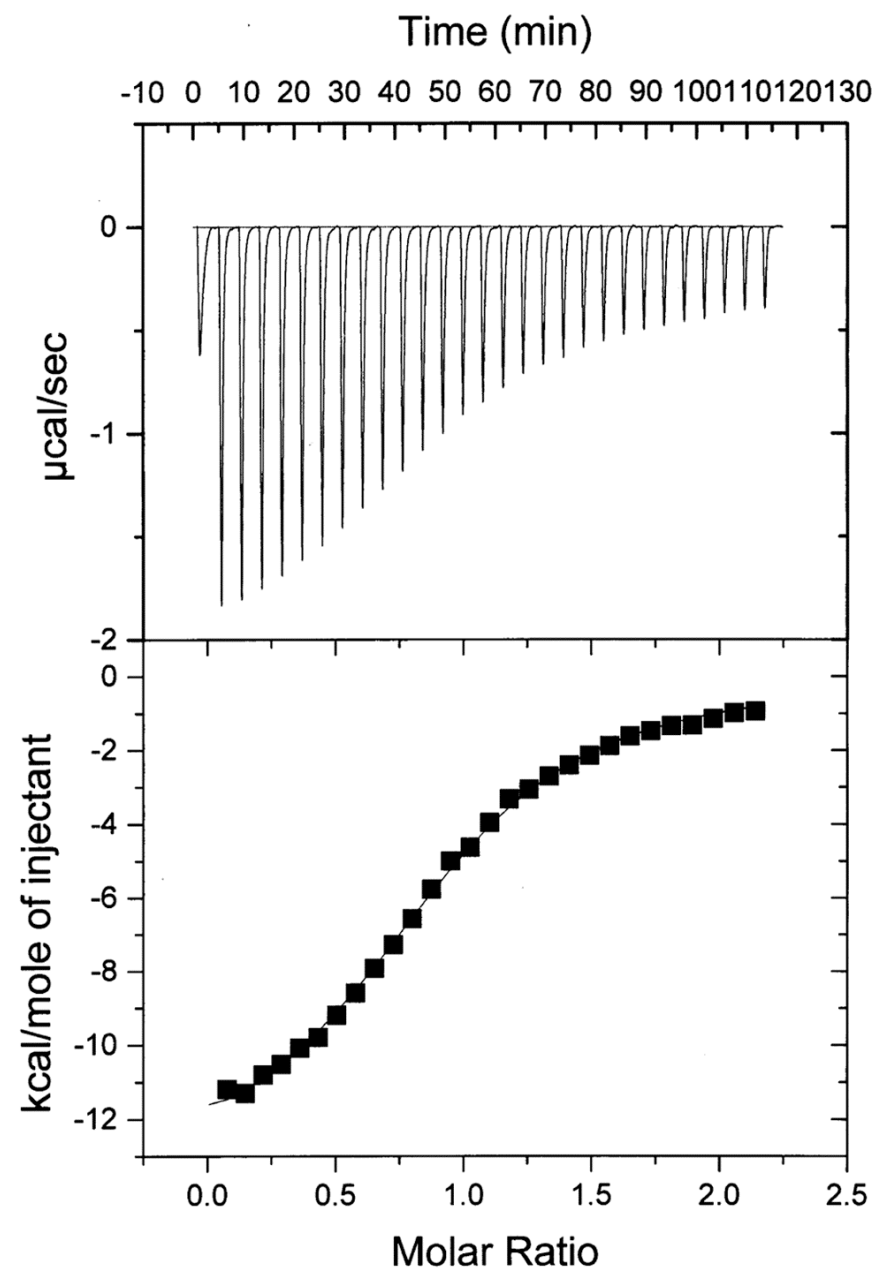


Figure 4.

A

B
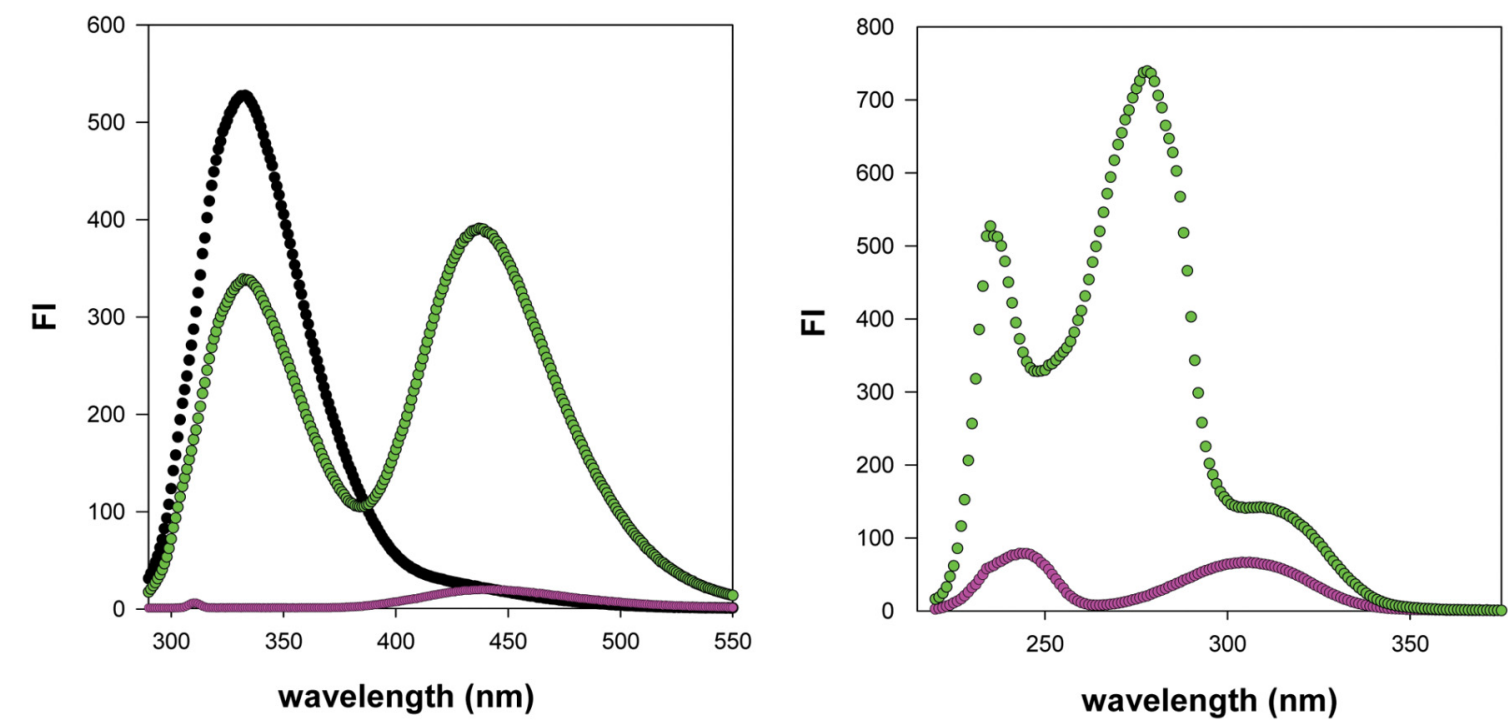

C

D
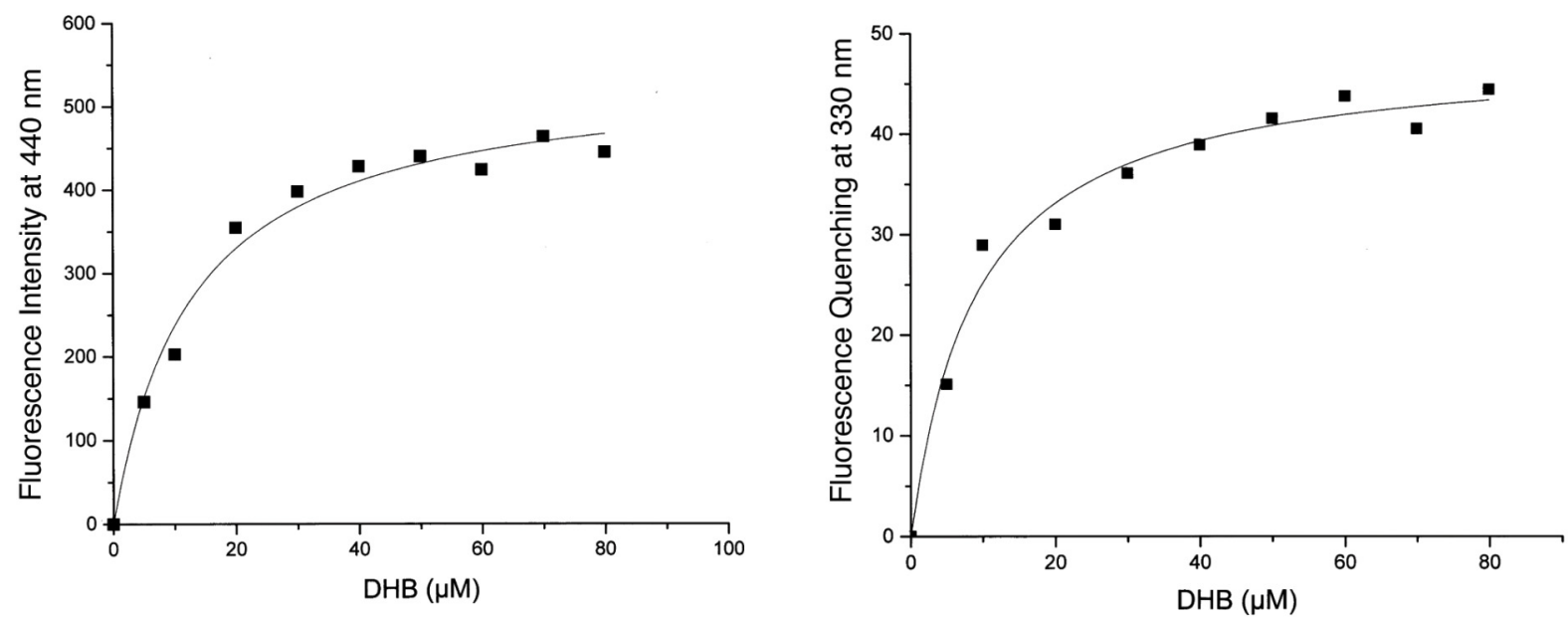
Figure 5.

A
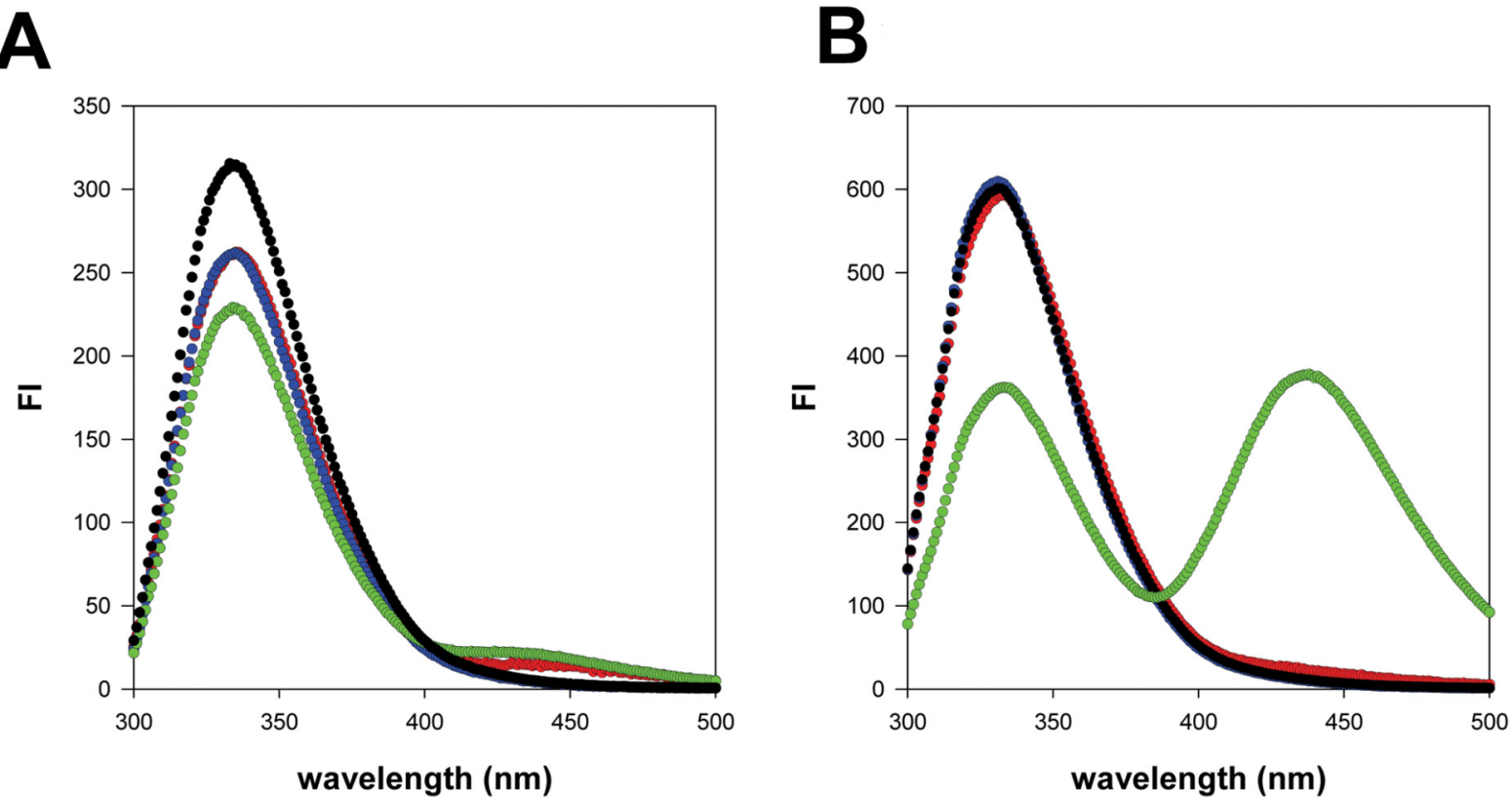
Figure 6.

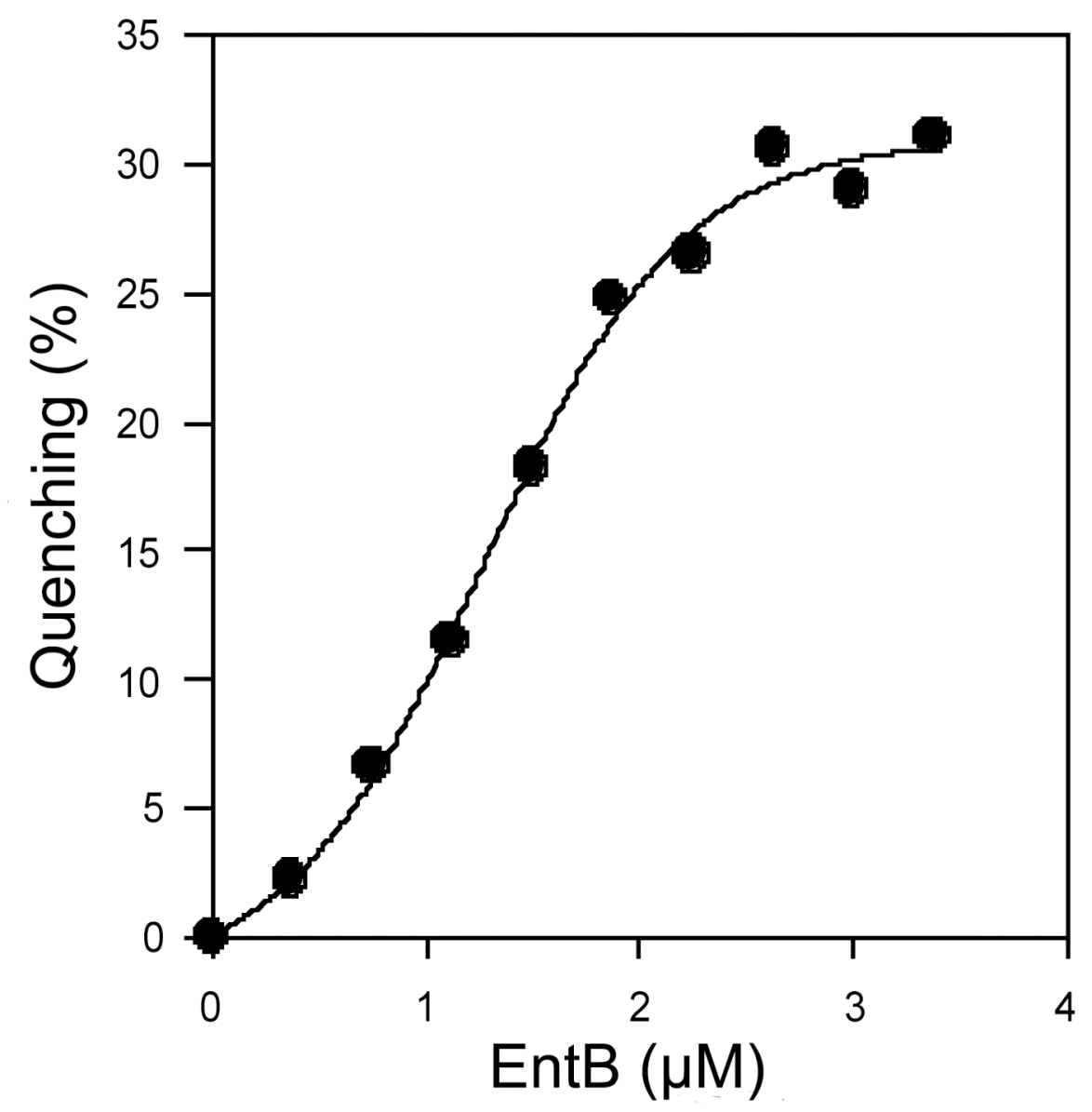


Figure 7.

\section{A}

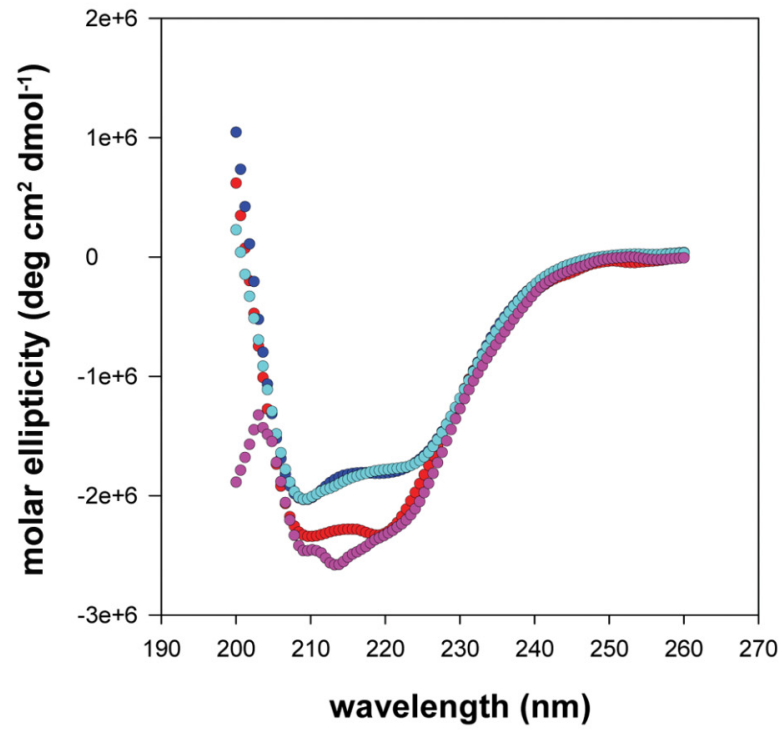

C

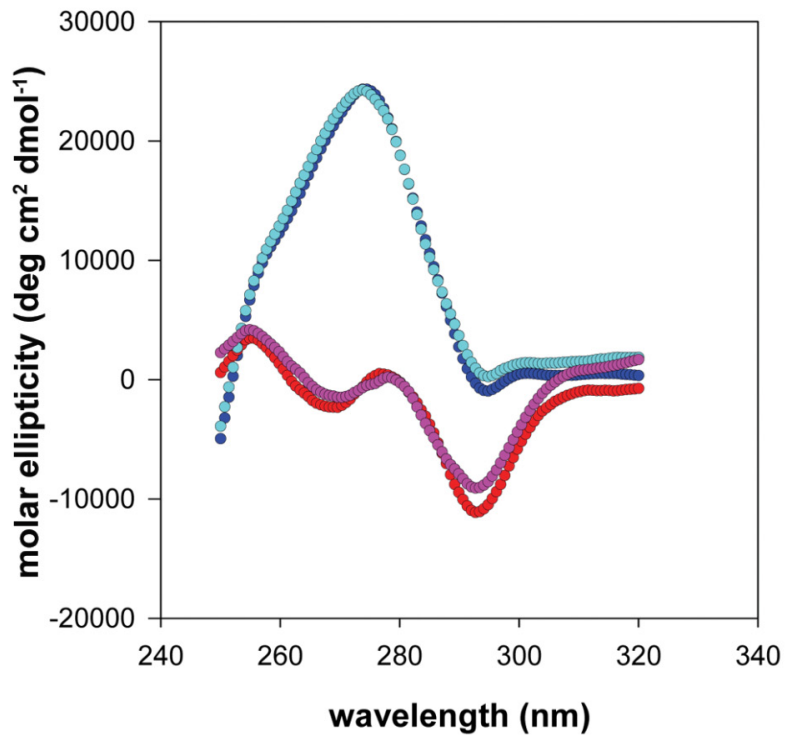

B

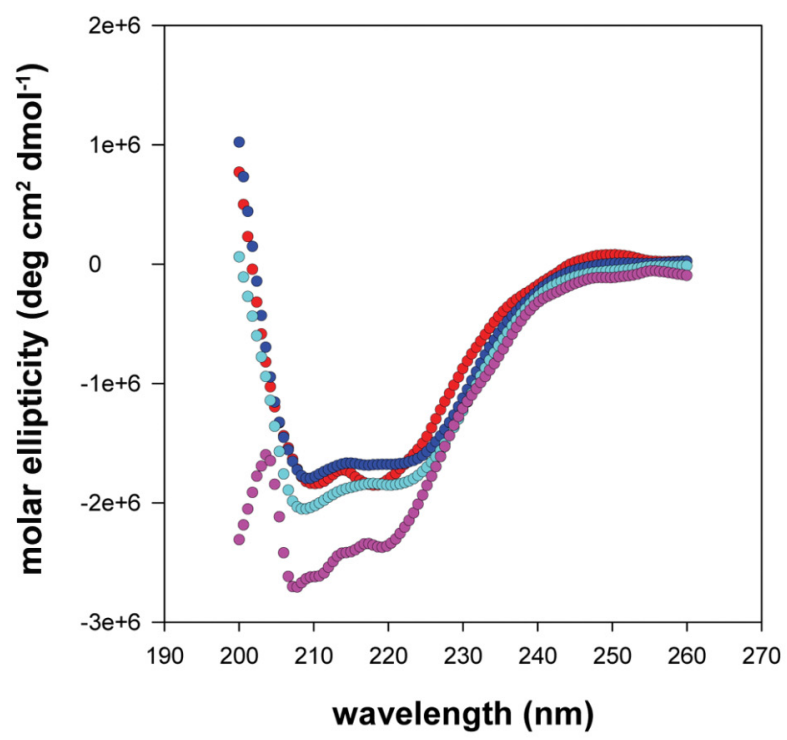

D

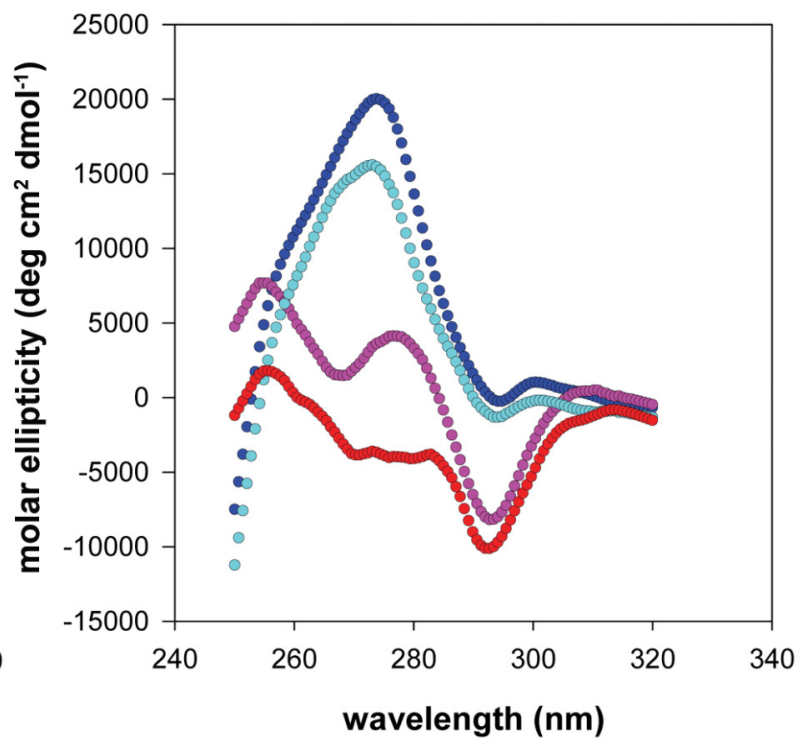


Figure 8.

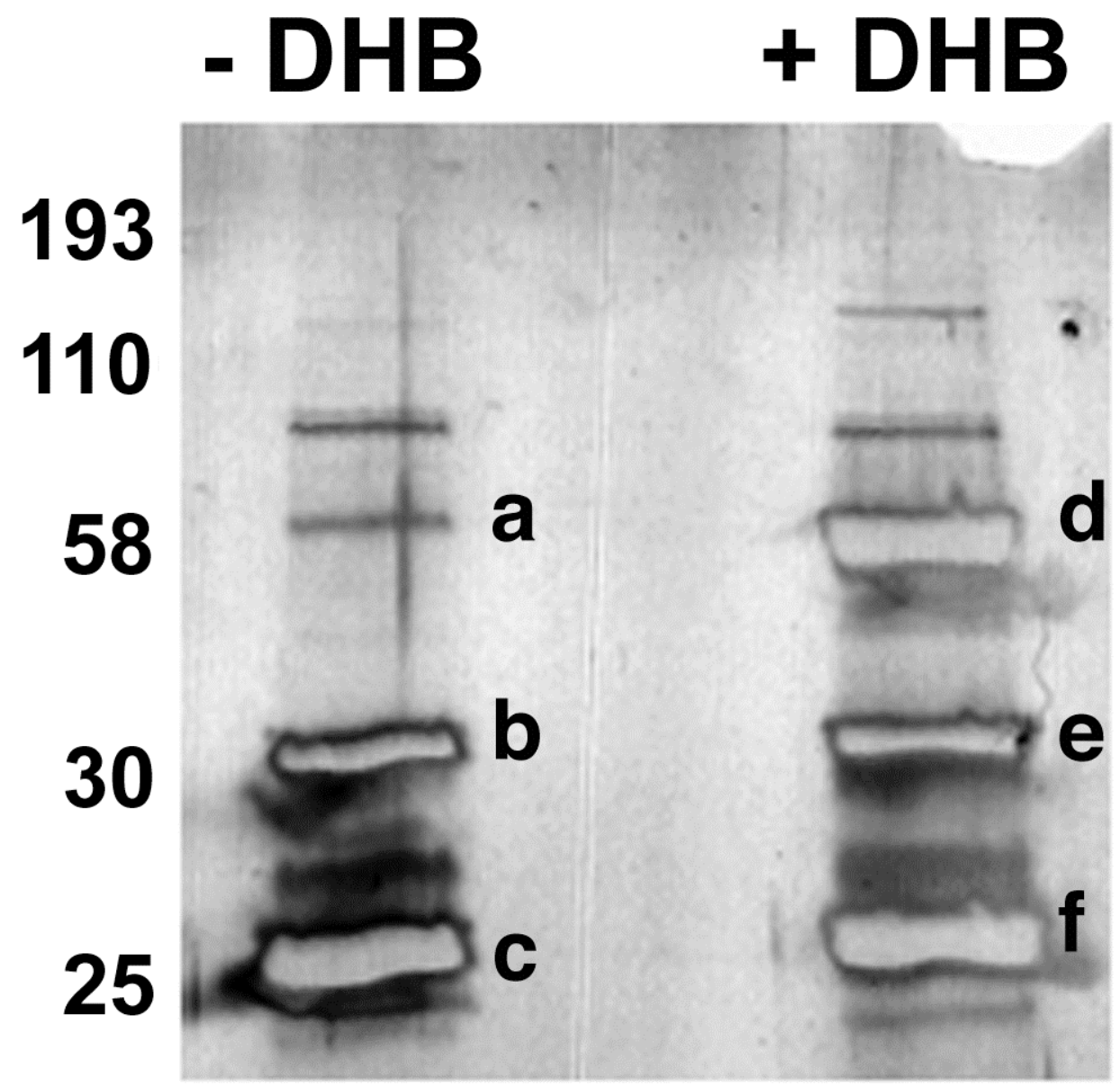

